Webbia

Journal of Plant Taxonomy and Geography

ISSN: 0083-7792 (Print) 2169-4060 (Online) Journal homepage: http://www.tandfonline.com/loi/tweb20

\title{
PALME DELL'INDO-CHINA
}

\section{ODOARDO BECCARI}

To cite this article: ODOARDO BECCARI (1910) PALME DELL'INDO-CHINA, Webbia, 3:1, 191-245, DOI: $10.1080 / 00837792.1910 .10669562$

To link to this article: http://dx.doi.org/10.1080/00837792.1910.10669562

曲 Published online: 12 Apr 2013.

Submit your article to this journal $2 \pi$

Џll Article views: 2

Q View related articles $₫$ 


\section{PALME DELL'INDO-CHINA PER ODOARDO BECCARI}

Avendo accettato l' incarico di elaborare le Palme per la Flora dell' Indo-China edita dal Prof. Lecomte, ed avendo per tale oggetto ricevuto in comunicazione il materiale relativo esistente negli Erbarî del " Muséum d' Histoire naturelle " di Parigi, ho creduto intanto opportuno pubblicare la dettagliata descrizione di quelle forme che mi son sembrate nuove per la Scienza. A quaste faccio seguire una enumerazione di tutte le Palme che sino a qui mi son note come indigene nell' Indo-China, nella speranza che ciò possa stimolare la raccolta di un più completo materiale di studio, essendo che molte delle Palme di detta regione sono rappresentate da esemplari cosi frammentarî, da riuscire di ben dubbia identificazione.

Io mi auguro quindi che con sollecitudine si effettuino nuove esplorazioni e raccolte, che completino quelle attualmente esistenti, in modo da render meno imperfetto il lavoro definitivo e sintetico sulle Palme Indo-Chinesi.

\section{Areca laosensis Becc. sp. n.}

Gracilis, caudice circiter $2.5 \mathrm{~cm}$. diam. Folia circiter metralia ; petiolo circ. $10 \mathrm{~cm}$. longo, $7-10 \mathrm{~mm}$. crasso, superne profunde canaliculato; segmentis paucis inter se approximatis, inaequalibus, 2-8-costulatis, lateralibus falcatoacuminatis, summis profunde inciso-dentatis. Spadices, circ. 25 cm. longi, basi densiuscule, superne laxiuscule 
lateque paniculati; ramis inferioris \pm divisis, superioribus simplicibus. Flores masculi secundi, gemini, $3 \mathrm{~mm}$. longi, trigoni, ovato-lanceolati, acuti ; staminibus 6 , antheris apice bidentatis; ovarî rudimento minutissimo. Flores foeminei 6-7 mm. longi, 4-4.5 mm. crassi, late ovati, obtusi. Fruıtus...

Descrizione. - Sembra una Palma un poco più gracile dell' Areca triandra, con un tronco, a giudicare dalla base di uno spadice, di circa, $2.5 \mathrm{~cm}$. di diam. Le foglie, dalle porzioni esistenti, si giudicano di circa $1 \mathrm{~m}$. di lunghezza; il picciolo, in un esemplare, è lungo $10 \mathrm{~cm}$. e spesso 7-10 mm., convesso di sotto, profondamente solcato o scavato a doccia di sopra, opaco $\theta$, sul secco, striato per il lungo. Il rachide è subtriangolare, convesso di sotto, bifaciale e con angolo acuto di sopra; i segmenti sono poco numerosi ed assai ravvicinati fra di loro: quelli presso la base sono lunghi $40 \mathrm{~cm}$., i superiori sono gradatamente più corti: di larghezza sono molto ineguali, alcuni (falciformi ed acuminati) hanno due sole costole primarie superiori; altri di dette costole ne hanno sino ad 8 ; i segmenti estremi (ultima e penultima coppia) formano un largo flabello forcato con tante profonde incisioni, corrispondenti a lobi più o meno falcati, quanti sono i nervi primarî; ogni lobo è alla sua volta più o meno profondamente fesso o bipartito.

Spadice similissimo a quello dell'Areca triandra, ma più gracile, lungo $25 \mathrm{~cm}$., ramoso sin quasi dalla base, a rami eretto-patenti; i rami inferiori sono un poco divisi, gli altri semplici; ogni ramo di solito porta alla base due fiori feminei ciascuno dei quali è accompagnato in alto da 2 fiori maschi, uno per parte, facilmente decidui; nel rimanente i ramoscelli sono più sottili che alla base, di circa $1 \mathrm{~mm}$. di spessore, e sono assai profondamente e fittamente scrobicolati da un sol lato, e portano in ogni scrobicolo 2 fiori maschi.

Fiori maschi lunghi circa $3 \mathrm{~mm}$., più o meno irregolarmente trigoni, ovato-lanceolati, acuti. Il calice è brevissimo ed è formato da 3 larghi denti triangolari acuti. Corolla 
divisa sino alla base in 4 segmenti lanceolati, acuti, rigidi, striati fittamente di fuori. Stami 6 con filamenti piuttosto brevi, ed antere bicornute o bidentate all'apice, a loggie profondamente bilobe in basso. Rudimento $d$ 'ovario minutissimo.

Fiori feminei latamente ovati, ottusi, lunghi 6-7 mm., larghi 4-4.5 mm., avvolti alla base da brattee larghissime e bassissime. Sepali larghi, ciliolati al margine. Petali di poco piủ lunghi dei sepali, molto larghi ed abbraccianti in basso, terminati da un piccolo apicolo inspessito che costituisce la sola parte nella quale i petali sono valvati.

I Frutti mancano.

Habitat. - Nel Laos meridionale a Phulet Phay nella regione ad oriente di Attopeu. Harmand, aprile 1877, n..$^{\circ} 1201$ nell' Erb. Pierre.

Osservazioni. - Specie imperfettamente conosciuta, dell'aspetto di una delle piccole forme della Areca triandra, ma decisamente distinta da questa per i suoi fiori maschi con 6 stami. Per questo motivo si avvicina alla $A$. oxycarpa di Celebes ed all' $A$. mammillata Becc. delle Filippine.

Pinanga baviensis Becc. sp. n.

Gracilis, caudice 1-1.5 m. alto, $13-15 \mathrm{~cm}$. diam.; foliis in segmenta pauca, late falcata, 5-7-costulata, acuminatissima partitis, et flabello profunde furcato terminatis; spadicibus simplicibus vel bipartitis, parte axili valde com. pressa; floribus masculis $5-6 \mathrm{~mm}$. longis, asymmetricis; calyce brevi, cupulari, trigono, truncato; petalis oblongolanceolatis, obtusis, minime falcatis; fructibus exacte bifariis, horizontalibus, olivaeformibus, $18-20 \mathrm{~mm}$. longis, $1 \mathrm{~cm}$. crassis, utrinque rotundatis ; semine oblongo, tereti, $14 \mathrm{~mm}$. longo, $8 \mathrm{~mm}$. crasso, basi truncato, ibique vix caudiculato, in vertice rotundato; rapheos ramis subparallelis, laxe et 
parcissime anastomosantibus, albumine in sectione transversa fere usque ad centrum radiato-ruminato.

Descrizione. - Gracile. Tronco alto 1-1.5 m., di 13-15 mm. di diam., con internodi lunghi $4-5 \mathrm{~cm}$. e leggermente clavati, minutamente marmorizzati da macchiette brunoporporescenti su fondo chiaro e con cicatrici delle foglie cadute annulari, larghe 2-4 mm. Fronde lunghe $80 \mathrm{~cm}$. (vista una sola), pinnatifide, con soli 3-4 segmenti per parte e terminate da un largo flabello, bipartito sino circa alla metà ; le divisioni del flabello hanno 10-12 costole primarie superiori e sono respettivamente larghe 8-10 cm., sono quasi orizzontalmente troncate all'apice e si terminano in tanti denti primarî triangolari, bidentati alla lor volta, quante sono le costole primarie; i denti primarî sono ottusi ed ottusamente bidentati; di questi gli intermedii

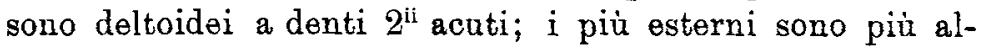
lungati degli intermedî ed i loro denti $2^{\text {ii }}$ sono acuminati; i segmenti laterali sono 5-7-costulati: sono falcati sin dalla base (che è molto larga), acuminatissimi, membranacei, opachi sulle due faccie, di sopra verdi e di sotto più pallidi, con le costole \pm forforacee, e sulle quali si trovano delle squamule decidue: i nervi terziarî sono numerosissimi, molto minutamente e fittamente papilloso-squamulosi. Picciolo lungo $10-11 \mathrm{~cm}$., spesso $7 \mathrm{~mm}$., raggrinzato sul secco, probabilmente crasso e subterete sul fresco; rachide pianeggante $\theta \pm$ squamuloso-leproso di sotto, con angolo molto acuto di sopra; vagina cilindrica, striata, squamuloso-macchiettata ccme il tronco; ligula brevissima (lunga 5-6 mm.), triangolare, acuta, opposta al picciolo.

Spadici reflessi subito dopo la fioritura, semplici od al più bipartiti, con parte peduncolare subterete e più o meno compressa, lunga $2-4 \mathrm{~cm}$., spessa $3-4 \mathrm{~mm}$.; spighette lunghe 11-14 cm., con parte assile fortemente compressa e leggermente sinuosa, larga $4 \mathrm{~mm}$, , $\theta$ portante sopra ognuno dei 2 lati 14-16 frutti, orizzontali e perfettamente bifarî; i pulvinuli sui quali riposano i frutti sono circolari, contornati 
da brattee angustissime; lo spazio interposto fra un pulvinulo e l'altro è di $4-5 \mathrm{~mm}$.

Fiori maschi asimmetrici, piccoli, lunghi 5-6 mm.; calice breve, cupolare, trigono, troncato alla bocca, attenuato in basso; petali irregolarmente oblunghi od oblungo-lanceolati (non falcati), ottusi e finamente striati.

Perianzio fruttifero cupolare, troncato, alto $2.5 \mathrm{~mm}$., di $5 \mathrm{~mm}$. di diam., con sepali e petali a contorno rotondato o molto poco distintamente apiculato nel centro.

Frutti oliveformi od ellittici, leggermente attenuati allo due estremità, molto minutamente mammillati all'apice, a base acuta, lunghi 18-20 mm., larghi $1 \mathrm{~cm}$. Seme oblungo, terete, lungo $14 \mathrm{~mm}$., largo $8 \mathrm{~mm}$., rotondato in alto, con la base tronca, pianeggiante e provvista di un brevissimo caudicolo; la superficio è segnata da numerose diramazioni del rafe, ascendenti e parallele, delle quali le centrali sca. valcano indivise l'apice e le altre si ripiegano sui lati e si anastomizzano leggermente sul lato opposto; albume compenetrato da 18-20 lamelle che giungono quasi sino al centro e che rendono il seme radiato-ruminato in sezione transversa.

Habitat. - Tonkino. Nelle Foreste del Monte Bavi, raccolta da B. Balansa il 22 genn. 1886 (n. 4372 nell' Erb. di Parigi).

Osservazioni. - Sembra alquanto affine alla Pinanga malayana, ma si distingue per le sue fronde con pochi $\theta$ larghi segmenti falcati e largo flabello forcato apicale; per i suoi spadici semplici, o con due sole spighe, ad asse fortemente compressa e coi fiori perfettamente biseriati; per i fiori maschi molto piccoli, relativamente alla maggioranza delle altre specie, e non falcati, ed infine per i frutti oliveformi ed il seme oblungo, troncato in basso con diramazioni del rafe quasi parallele $\theta$ poco anastomosate, che corrispondono ad altrettante lamelle (circa 18) che penetrano nell'albume e lo rendono radiato-ruminato, allorchè 
viene osservato in sezione transversa. Appartiene alla sezione Orthostichanthae.

Dalle altre due specie note della Cocincina (P. Cochinchinensis Bl. \& P. Duperreana Pierre) subito si distingue per $\mathrm{i}$ suoi spadici semplici od al più biforcati, $\theta$ per $\mathrm{i}$ fiori $\delta$ non falcati, con calice a denti corti; nelle due ora menzionate specie, gli spadici hanno varî rami, ed i fiori $\sigma$ sono falcato-acuminati.

\section{Caryota monostachya Becc. sp. n.}

Parva, gracilis, caudice cir. $1 \mathrm{~m}$. alto, $2.5-3 \mathrm{~cm}$. diam. ; frondibus simpliciter duplicato-pinnatis, pinnis late elongatocuneatis, $12-18 \mathrm{~cm}$. longis, $6-8 \mathrm{~cm}$. latis, apice oblique truncatis et ondulato-praemorsis; spadicibus simplicibus, recurvo-pendulis, elongatis; spica crassa, $30-60 \mathrm{~cm}$. longa, parte pedicellari erecta elongata, digiti minoris crassitiae, suffulta; spathis vaginantibus, superioribus auriculato-elongatis; floribus masculis ovato-oblongis, apice conico obtusiusculo, majoribus $17 \mathrm{~mm}$. longis, $8 \mathrm{~mm}$. crassis; calyce cupulari truncato; corolla calyce 3-plo longiori; staminibus numerosissimis; fructibus majusculis, sphaericis vel paullo depressis: majoribus usque ad $3 \mathrm{~cm}$. diam., dispermis; seminibus dimidiato-sphaericis, 17-23 mm. latis, 18-21 mm. longis; 10-12 mm. crassis, superficialissime ruminatis.

Descriziona. - Fra tutte le sin qui note è la più piccola specie del genere. Il tronco ha circa l'altezza di $1 \mathrm{~m}$. (Balansa), e sembra che non abbia più di $2.5-3 \mathrm{~cm}$. di diam. allorchè è inguainato dalle basi delle foglie. Gli spadici sono indivisi; di essi da prima ne comparisce uno termịnale e poi successivamente varî altri laterali (Balansa).

Foglie semplicemente duplicato-pinnate, ossia il rachide principale porta a destra ed a sinistra dei rachidi secondarî 
(o pinne primarie) sui quali si trovano i segmenti (in tutte le altre specie $i$ rachidi secondarî sono divisi in rachidi terziarî); guaina fessa lungo il lato ventrale, con i margini guarniti di poche fibre nere finissime; picciolo assai lungo, subterete, di $12 \mathrm{~mm}$. di diam., con un superficialissimo accenno di solco sulla faccia superiore; il rachide nella parte intermedia $\dot{e}$ rotondato di sotto, ed ha uno spigolo prominente ottuso lungo il centro, ed un solco per parte, dove sono inseriti i rachidi secondarî; i margini sono ottusi; le pinne primarie sono lunghe $50-70 \mathrm{~cm}$. (le inferiori più lunghe delle superiori), inserite ad un angolo assai acuto; quelle più basse hanno 5-6 segmenti per parte cltre quello terminale; quelle della parte intermedia ne hanno solo 2-4; il rachide delle pinne $\dot{\theta}$ compresso lateralmente, con spigolo ottuso di sotto, ed è percorso da uno strettissimo solco di sopra.

I segmenti sono cartacei, verdi sopra ambedue le faccie, ma leggermente più pallidi di sotto; i basilari di ogni pinna (quelli almeno delle pinne intermedie) sono più grandi e più larghi dei superiori, ineguali, latamente cuneati, col margine superiore ondulato-sinuoso, dentato-premorso, prolungato in punta lungo il margine esterno; sono larghi $6-8$ cm., lunghi 12-18 cm.; i segmenti laterali sono inseriti ad un angolo molto acuto, sono assai più stretti dei basilari $\theta$ lungamente cuneati verso la base che è acuta, e sono prolungati superiormente in una assai lunga coda sottile lungo il margine inferiore, mentre il superiore è molto più corto, di guisa che il margine anteriore od apicale, che è ondulato e premorso, risulta obliquissimo; i segmenti risultano perció lunghi e stretti (lunghi $18-20 \mathrm{~cm}$. e larghi 3-4 cm.); il segmento terminale è il più grande di tutti, $\dot{\theta}$ allungato ed attenuato verso l'apice ed ha il contorno più o meno lobato.

Spadici semplici, consistenti in una sola e grande spiga arcuata in basso e poi pendente, lunga in media nella parte fiorifera $40-50 \mathrm{~cm}$., ed altre volte solo 30 od anche sino $60 \mathrm{sm}$., essa ha circa $7 \mathrm{~mm}$. di spessore, è portata da 
una parte pedunculare eretta, grossa al più, quando è vaginata dalle spate, quanto il dito mignolo, e lunga quanto la parte fiorifera od anche assai meno; questa parte peduncolare $\dot{e}$ vaginata da 3-4 spate allungate, forforacee esternamente, glabre, più scure e striate di dentro; le più basse tubulari, fesse in alto da un lato; le superiori gra. datamente più lunghe con breve parte tubulare nella parte pit bassa e con lembo aperto e strettamente lanceolatoauriculare, acuminato, nel rimanente; la spata superiore abbraccia la base della parte fiorifera.

Fiori in glomeruli molto ravvicinati, al solito modo terni, e con i fiori maschili precoci; quando questi sono in boccio completamente sviluppato i feminei sono latamente conici, lunghi circa $7 \mathrm{~mm}$., prossimi a raggiungere il loro completo sviluppo, ed hanno la corolla di già sporgente dal calice.

Fiori maschili ovato-oblunghi, leggermente ristretti verso l'apice in punta conica ottusa; sono a quanto sembra assai variabili di dimensioni indipendentemente dal loro grado di sviluppo; al momento dell'antesi sono lunghi sino $17 \mathrm{~mm}$. e spessi 8; alle volte però non raggiungono queste dimensioni. Calice cupolare, troncato; sepali-reniformi, a contorno spesso crenulato, lisci all'esterno, gibbosi in basso. Corolla circa 3 volte più lunga del calice; petali coriacei, navicularioblunghi, acutiusculi, finamente striati all'esterno sul secco. Stami numerosissimi (60 in un fiore); antere sessili o quasi, strettamente lineari, lunghe 9-10 mm., acutiuscule; rudimento d'ovario inconspicuo.

Fiori feminei accolti framezzo a 2 conspicue brattee reniformi a contorno rotondato, crenulato, grandi quanto $i$ sepali del fore femineo: sono conici, larghi alla base 5 mm.; sepali suborbicolari, a contorno rotondato intiero o più o meno crenulato e minutissimamente ciliolato, rotondati e lisci sul dorso, finamente striato-nervosi internamente; corolla circa il doppio più lunga del calice, divisa per almeno $i$ due terzi in 3 segmenti deltoidei acuti; ovario globoso, ottusamente 3-lobo, con due loggie; talvolta 
oltre l'ovario biloculare s' incontra una carpella rudimentaria staccata; stigmi due, carnosi, triangolari, acuti, poi patenti; talvolta esistono 3 staminodî filiformi sottilissimi, con antera rudimentaria lineare subulata, inseriti nei seni fra i segmenti della corolla e lunghi quanto questi; gli staminodî possono però mancare tutti od esser presenti solo 1-2.

Frutti fra i più grandi nel genere, sferici, molto leggermente depressi, ma non incavati in alto, con $i$ resti degli stigmi formanti una piccola cicatrice triangolare a bocca di mignatta; essi sono assai variabili di grandezza (i maggiori misurano sino $3 \mathrm{~cm}$. di diam.), hanno la superficio unita, nera sul secco, opaca, non perfettamente liscia, ma nemmeno granulosa; di solito contengono 2 semi. Pericarpio molto sottile e fragile sul secco, a quanto sembra con mesocarpio non carnoso e scarsissimo. Semi piano-convessi o dimidiato-sferici, spesso più larghi che lunghi, a testa opaca, nerastra, che parzialmente si distacca in forma di pellicole fragili: sono larghi 17-23 mm., lunghi 18-21 mm., spessi 10-12 mm.; diramazioni del rafe numerose, verticali, corrispondenti alle impressioni esterne della superficie del seme, poco anastomosate, tutte convergenti in un sol punto sul lato dorsale presso l'apice. L'albume è molto superficialmente ruminato dalle intrusioni del tegumento esterno che penetrano solo $1-3 \mathrm{~mm}$. nel suo interno, e che corrispondono alle impressioni della superficie esterna del seme.

Habitat. - Nel Tonkino occidentale a Thien Thòn: Père Bon (29 genn. 1892, n. ${ }^{0} 5046$ nell'Erb. di Parigi). -Pure nel Tonkino, nelle foreste del Monte Bavi (22 luglio 1886) e nella China meridionale nel Kouy-Tcheou, raccolta da Cavalerie e Fortunat, con l'indicazione: * Route Lo-fou-Lo-Kueus, sur pente abrupte $\gg\left(\right.$ n. $^{0}$ 2645, Erb. di Parigi).

Osservazioni. - Specie distintissima fra tutte le conosciute per le sue piccole dimensioni, per le fronde solo 
duplicato-pinnate, ma sopratutto per gli spadici con una sola spiga, grossa come una di quelle dello spadice ramoso della Caryota Rumphiana e con frutti anche più grossi che in questa specie.

Forma il passaggio fra le Caryota ed Didymosperma; si distingue poi da tutte le altre specie di Caryota sino a qui note, per i suoi semi molto superficialmente ruminati, le anguste intrusioni dell'integumento del seme non penetrando nella massa dell'albume che per il tratto di $1-3 \mathrm{~mm}$.

Didymosperma caudata Wendl. et Drude in Kerch. Les Palm. 243 ; Beccari, Malesia, III, 96. - Wallichia caudata Mart. Hist. Nat. Palm. III, 315. - Borassus caudata Lour. Fl. Coch. II, 619 ; edit. Willd. II, 760 .

Specie estremamente polimorfa, della quale si possono distinguere almeno 3 forme principali, che a prima vista si potrebbero ritenere quali altrettante specie distinte, ma che in causa delle forme di transizione non è possibile definire con caratteri diagnostici precisi. Presa nel suo insieme la Didymosperma caudata sembra una palma assai diffusa in tutta l'Indo-China. La forma del Tonkino potrebbe forse considerarsi come specie distinta per le sue piccole dimensioni e per qualche particolarità del fiore.

Tanto nella forma tipica della Didymosperma caudata, quanto nelle sue varieti, il frutto di solito contiene 3 semi bene sviluppati, sebbene si trovino anche frutti con due od anche con un sol seme. Non è quindi un carattere costante per le Didymosperma quello di avere un frutto con soli due semi; $\theta$ mancando questo carattere non esiste alcun mezzo per distinguere questi dalle Arenga ad eccezione delle piccole dimensioni dei rappresentanti del primo genere. Una certa differenza sembrava che esistesse nella forma dei segmenti delle foglie, essendo che quelli della Arenga sono sempre più o meno lineari, mentre tutte le 
Didymosperma finora conosciute avevano segmenti più o meno romboidali-cuneiformi, ossia larghi in alto ed attenuati più o meno lungamente verso una base acuta. Ma adesso si presenta una singolarissima varietà della $D$. caudata (stenophylla) nella quale i segmenti sono lineari con denti scalati esattamente come in una tipica Arenga. Che questa ultima natura di segmenti rappresentino una forma di transizione da quella cuneata è dimostrato in modo indiscutibile dalle forme di passaggio che collegano la varietà con il tipo.

Dallo studio molto accurato che ho eseguito sopra un gran numero di esemplari si possono distinguere le 3 seguenti forme principali della Didymosperma caudata.

Didymosperma caudata Wend. et Drude (forma typica).

Palma gracilis, 1-2 m. alta, caudice vaginato digiti minoris crassitie. Folia interdum metralia et ultra, vulgo 40-50 $\mathrm{cm}$. longa, simpliciter pinnatisecta, segmentis utrinque solitariis vel basilaribus saepe geminatis, elongato-cuneatis vel subrhombeo-cuneatis, in margine anteriore praemorsodentatis et apice in medio caudatis, majoribus $15-25 \mathrm{~cm}$. longis, superne $7-8 \mathrm{~cm}$. latis, segmento terminali usque ad basin tripartito vel 3-costulato et plus minusve 3-lobo. Spadices simplices, elongati, nutantes vel plus minusve cernui, vulgo $30-60 \mathrm{~cm}$. longi, in torum dimidiam superiorem partem floriferi; parte pedunculari spathis tubulosis arcte vaginantibus induta. Flores glomerulato-terni, foeminei serotini; flores masculi anguste ovato-elliptici vel subclavato-elliptici, acuti, 5-7 $\mathrm{mm}$. longi, 2-5 mm. lati. Flores foeminei globosi, circiter $5 \mathrm{~mm}$. diam. Fructus globoso-ovati, 10-13 mm. longi, 9.5-10.5 mm. diam.; seminibus vulgo 3 , obtuse trigonis.

Descrizione. - Gracilissima. Fusto alto al più circa $1 \mathrm{~m}$, allorchè vaginato grosso al più quanto un dito rnignolo, 
ma di solito di soli 5-7 mm. di diam. Foglie molto variabili anche nella pianta adulta e sul medesimo fusto; in uno esemplare molto robusto una è lunga $90 \mathrm{~cm}$., di cui circa 25 ne misura la parte picciolare, altre sono solo 40-50 $\mathrm{cm}$; le foglie presso l'apice del fusto fertile sono più corte di quelle più basse; tutte hanno pochissimi segmenti (da 2-3 a 6-7 per parte) più uno terminale; guaina cilindrica, dilacerato-fibrosa lungo it lato ventrale, specialmente in alto e presso la bocca; ligula ocreiforme, allungata (lunga 5-6 cm.) sottilmente membranacea, marcescente e presto risoluta in fibre brune sottilissime; picciolo di lunghezza molto variabile sulla stessa pianta (nelle fronde basse il picciolo è più lungo che nelle alte), subterete, di 3-6 mm. di spessore, striato per il lungo: nelle fronde di recente svolte è più o meno corrugato-angoloso (negli esemplari d'erbario), opaco, fugacemente forforaceo-rubiginoso, poi minutamente punteggiato-scabridulo; rachide subtrigono. Segmenti di solito solitarî in alto, e geminati sopra ogni lato nella parte bassa del rachide; talvolta quasi tutti geminati; le coppie od i solitarî di un lato spessissimo (ma non sempre) opposti a quelli dell'altro lato; essi sono \pm elongatocuneati, o cuneato-subromboidali, molto variabili riguardo alle dimensioni ; i maggiori sono lunghi $15-22 \mathrm{~cm}$., e larghi $7-8 \mathrm{~cm}$; altri però sono larghi solo $2-3 \mathrm{~cm}$., ma non più corti; nelle forme più grandi sono cuneati nei due terzi inferiori $\theta$ quasi simmetricamente trigono-cuspidati nel terzo apicale; più raramente sono asimmetrici e con il margine anteriore obliquo, ed in questo caso con la costola mediana eccentrica ed una costola più gracile da una parte; in generale hanno la parte centrale prolungata in una assai lunga punta caudiforme, sono ondulati $\theta$ denticolato-premorsi su tutto il margine anteriore. Il segmento apicale è più grande dei laterali, $\dot{\theta}$ cuneato-flabelliforme, 3-5-costulato, a contorno con tanti lobi acuti e caudati quante sono le costole; essi segmenti sono cartacei, rigiduli, verdi e quasi nitenti di sopra, più pallidi e nelle fronde giovani distintamente albicanti e molto tenuamente 
forforacei di sotto; alla fine rimangono anche glabri, ma allora appariscono minutamente e fittamente punteggiati, $i$ puntini risultando dalle basi dei tricomi caduti: hanno la costola mediana sottile, prominente e rotondata di sotto, superficiale di sopra, sono striati sulle due faccie da un numero variabile di nervi secondarî e da numerosi nervi terziarî.

Spadici terminali e laterali, uscenti solitarî, o talvolta in numero di $2-3$, dal lato ventrale in alto, da ognuna delle guaine delle fronde superiori; essi sono glabri in ogni parte, semplicissimi, indivisi, filiformi, nutanti e risultano costituiti da una sola spiga fiorifera, lunga $10-25 \mathrm{~cm}$., portata da una parte peduncolare lunga circa quanto la spiga, od anche alquanto più corta, ma sempre vaginata per intiero, o quasi, da varie spate più o meno distanziate, di cui le primissime o più basse brevi, quasi squameformi e le seguenti tubulari, assai strettamente guainanti in basso, aperte dal lato ventrale verso l'alto; le spate superiori, e specialmente l'ultima, sono prolungate \pm in un lembo papiraceo, strettissimamente auriculeforme, acuminato; la parte peduncolare dello spadice, allorchè è inguainata dalle spate, è di 4-5 mm. di diam., il nudo asse è di $1-3 \mathrm{~mm}$. di diam.; l'asse sembra che da prima, quando porta solo fiori $\delta$, sia più sottile e che in seguito ingrossi quando porta i fiori $q$ bene sviluppati, e più specialmente poi al momento della maturità dei frutti; la parte fiorifera non è sensibilmente più spessa della pedunculare, è glabra, corrugato-striata, e molto leggermente sinuosa fra i glomeruli dei fiori, quando questi non sono molto ravvicinati. Gli spadici sono tutti monoici, ma al momento che i fiori maschi sono perfettamente sviluppati, $i$ fiori feminei non si vedono e rimangono ancora allo stato primordiale, accolti fra due brattee strettamente combacianti fra loro e situate framezzo ai fiori maschili.

I fiori maschili in boccio sono molto angustamente ellittici, o subclavato-ellittici, lunghi 5-7 mm., larghi $2.5 \mathrm{~mm}$., acuti; calice cupolare, troncato; sepali suborbicolari, poco 
più larghi che lunghi, a contorno rotondato, intiero, carnoso-gibbosi o calcarati alla base; corolla tre volte, od anche un poco più, più lunga del calice, divisa sino in basso in 3 segmenti naviculari, lanceolato-oblunghi, acuti, finamente striati all'esterno; stami circa 25; antere lineari, angustissime, attenuate un poco alle due estremità, quasi acute alla base e più o meno anche all'apice od anche ottuse, erette sopra un sottilissimo filamento assai lungo, ma più corto dell'antera e che si inserisce in una leggiera smangiatura della loro base; manca qualunque rudimento d'ovario.

Fiori feminei sviluppantesi assai dopo la caduta dei fiori maschili, orizzontali, inseriti spiralmente ed assai regolarmente intorno l'asse, che ricuoprono piuttosto densamente; sono globosi, di circa $5 \mathrm{~mm}$. di diam.; brattee florali reniformi, sepaloidee, fortemente striate; calice spianato con sepali suborbicolari ma più larghi che lunghi, a contorno rotondato, finamente ciliolato, fortemente striato-nervosi; corolla 2 volte più lunga del calice, divisa sino quasi alla base in 3 segmenti concavi, larghi, subdeltoidei, ottusiusculi ed abbraccianti l'ovario, fortemente striato-nervosi. Staminodî 0. Ovario globoso, leggermente depresso, triloculare, rotondato in alto, ed ivi con un accenno di 3 superficialissime carene radianti dagli stigmi; questi sono sessili, trigoni, conniventi e formano con la loro riunione una piccola areola circolare piana.

Frutti globosi o globoso-ovati, rotondati alle due estre. mità, con la piccola areola stigmatica apicale rotonda, piana ed immutata, senza fessure od incavi e con appena un accenno di 3 carene radianti dal contorno dell'areola; la superficie è opaca ed unita; la forma varia, ma è sempre più o meno globosa, ed il diametro oscilla fra i 9.5-10.5 mm.; il pericarpio $\dot{e}$ sottile, crostaceo, essucco (forse a perfetta maturita $\dot{e}$ leggermente carnoso). Semi di solito 3, talvolta 1-2; quando sono 3 risultano convessi dal lato esterno e con due faccette dal lato interno: allorchè ben maturi sono quasi neri a superficie nitida, segnati 
da poche diramazioni del rafe ascendenti dalla base dal lato interno e pochissimo ramose; albume osseo, omogeneo; embrione situato verso la metà del lato convesso.

Habitat. - Assai frequente in tutta l'Indo-China. Nella Cocincina meridionale a Baochiang, prov. di Bien-hoa: Pierre $\mathrm{n}^{\circ} 1877$ (a questo numero è unita la nota: erecta vel subscandens); un secondo esemplare, pure col n. ${ }^{\circ} 1877$, ̀̀ dei Monti Keerev nel Camboge. Ad un altro esemplare Pierre assegna il nome annamita di "Mâj-roi » (rotin fouet). Il Dott. Thorel ha raccolta questa forma durante la spedizione del Me-kong (1866-68) a Bassac ( . $^{\circ}$ 2375), a Kemarath (n." 2992) ed a Lawang-Prabang (senza numero). Nel Laos a Phon thane: Spire n. 92 (Esemplari tutti dell' Erb. di Parigi).

Osservazioni. - I frutti variano assai per la forma e quindi anche la forma del seme varia. Negli esemplari di Pierre n. 1877 di Baochiang i frutti hanno 3 semi (1-3 secondo nota di Pierre), sono di $10 \mathrm{~mm}$. di diam., ma un poco più larghi che lunghi. Il seme perfettamente maturo si libera facilmente dagli invogli ed ha una superficie nitida color castagno scuro; ha le faccie ventrali separate da un angolo ottuso, $\dot{e}$ rotondato alle due estremità ed $\dot{e}$ lungo $6 \mathrm{~mm}$., largo $5.5 \mathrm{~mm}$., ed ha $4 \mathrm{~mm}$. di spessore.

$\mathrm{Nel} \mathrm{n}^{\circ} 2375 \mathrm{di}$ Thorel il frutto (pure con 3 semi) è ovato, lungo $13 \mathrm{~mm}$. e largo $9.5-10 \mathrm{~mm}$.; il seme è piuttosto acuto alle due estremità ed é lungo $9 \mathrm{~mm}$. ed ha la superficie opaca, ma non è perfettamente maturo.

Nel n. ${ }^{\circ} 92$ di Spire, di Phon thane, il frutto contiene 2-3 semi ed è sferico, di 9.5-10.5 mm. di diam.; il seme che è bene sviluppato $\theta$ sembra completamente maturo, è lungo 8 mm., largo $6 \mathrm{~mm}$. e spesso $4 \mathrm{~mm}$.

Per quel che riguarda la forma dei segmenti oltre alla varietà stenophylla, qui appresso descritta, si potrebbe stabilire una forma intermedia. Vi è infatti un esemplare fra quelli del Dott. Thorel, raccolto a Lacone che ha alcuni 
segmenti lunghi $20-25 \mathrm{~cm}$. e larghi al di sopra della metà o verso il terzo superiore solo $15 \mathrm{~mm}$., e da questo punto cuneati in basso, e nella parte superiore ristretti a scalini, e denticolati; il segmento centrale $̇$ 3-costulato e con 3 lobi acuti. Un altro esemplare (di Kemarath, pure del Dott. Thorel), ha taluni segmenti un poco più larghi e più distintamente cuneiformi del precedente, ma sempre più stretti che nella forma tipica (lunghi $12-22 \mathrm{~cm}$. e larghi solo $2-3 \mathrm{~cm}$.).

\section{Didymosperma caudata var. stenophylla Becc.}

Frondium segmentis linearibus, $20-28 \mathrm{~cm}$. longis, $6-7 \mathrm{~mm}$. latis, 3 terminalibus liberis, lateralibus simillibus, marginibus remotissime dentatis et per gradus decrescentibus, apice obtuso acute denticulato; caetera uti in forma typica.

Descrizione. - Non sono conosciute le dimensioni generali, ma dalle porzioni esistenti apparisce una pianta gracilissima $\theta$ probabilmente non più alta di 1 metro, essendo il tronco inguainato di soli 5-6 $\mathrm{mm}$. di diam.

Foglie di pianta fertile lunghe, sullo stesso fusto, da 40-60 cm., con pochissimi segmenti, \pm 6 per parte ed uno terminale; guaina cilindrica, finamente striata per il lungo, leggermente dilacerato-fibrosa in alto sul lato ventrale $\Theta$ specialmente presso la bocca; fibre bruno scure; ligula allungata, acuminata, guainante in basso, molto sottilmente membranacea, marcescente e presto risoluta in fibre brune sottilissime; picciolo di lunghezza molto variabile sulla stessa pianta ; nelle fronde basse caulinari è, a quanto sembra, più allungato, $(17 \mathrm{~cm}$. in un esemplare) che nelle superiori (4-6 cm.), è subterete, striato per il lungo, opaco, fugacemente forforaceo-rubiginoso; rachide subtrigono. Segmenti cartacei, rigiduli, nella parte bassa del rachide geminati sopra ogni lato; le coppie di un lato opposte a quelle dell'altro lato; i segmenti superiori sono solitarî ed opposti; 
tutti sono molto strettamente lineari; attenuati verso una base acuta, a margini intieri meno che presso l'apice, dove sono ristretti a scalini in punta più o meno lunga e per lo più ottusa, e quivi assai acutamente denticolati; sono lunghi 20-28 cm., larghi 6-7 mm. La foglia si termina in 3 segmenti liberi, di cui il centrale poco differisce dagli altri ; sono verdi e quasi nitenti di sopra, più pallidi o subalbicanti $\theta$ molto tenuamente forforacei di sotto, hanno la costola mediana sottile, prominula e rotondata di sotto, superficiale di sopra, striata sulle due faccie da alcuni nervi secondarî e da numerosi nervi terziarî.

Spadici terminali e laterali, uscenti solitarî da ognuna delle fronde superiori, semplicissimi, indivisi, filiformi, nu. tanti, consistenti in una sola spiga lunga $15-25 \mathrm{~cm}$. e portata da una parte pedunculare un poco più corta di lei; del resto esattamente come nella forma tipica.

Habitat. - Indo-China. Raccolta a Vien-chang dal Dott. Thorel durante la spedizione del Me-kong (1866-68).

Osservaziont. - Differisce dalla forma tipica solo per i segmenti strettissimi, lineari, che in piccolo hanno esattamente la forma di quelli dell'Arenga saccharifera. Gli spadici con $i$ fiori feminei sono esattamente come nella forma tipica; di quelli maschili non ne ho visti.

L'unico carattere che potrebbe servire a distinguere i Didymosperma dalle Arenga, quello della forma dei segmenti, manca in questa varielà, che sembra proprio formare il passaggio fra i due rammentati generi. L'ovario triloculare piuttosto che biloculare sembra abbia poco importanza come carattere generico in questo gruppo di Palme. Il curioso di questa Palma si è quindi che la forma tipica sarebbe un Didymosperma e la varietá una Arenga. 


\section{Didymosperma caudata var. tonkinensis Becc.}

Caudice gracili, brevi; frondium segmentis rhombeo-cuneatis; spadicibus brevibus rigidis; floribus masculis obovatis, in vertice rotundatis.

Descrizione. - Palma gracilissima, stolonifera, alta 0.50-1 m. Fusto, allorchè coperto dalle guaine, di $5-7 \mathrm{~mm}$. di diam.; il fusto nudo solo 4-5 mm.

Foglie piccole, variabili di lunghezza da $25-45 \mathrm{~cm}$. anche sulla medesima pianta; tutte hanno pochissimi segmenti, solo 1-3 per parte, più uno terminale; guaina cilindrica, fortemente striata, dilacerato-fibrosa in alto lungo il lato ventrale, specialmente in alto presso la bocca; ligula ocreiforme, allungata, molto sottilmente membranacea, marcescente e prestissimo risoluta in fibre brune tenuissime; picciolo di lunghezza molto variabile, lungo da 2-3 a $15-20 \mathrm{~cm}$. sulla stessa pianta, fortemente striato (sul secco), subterete od oscuramente angoloso, \pm distintamente canaliculato nella faccir superiore, di $2-3 \mathrm{~mm}$. di spessore, leggermente forforaceo da principio, poi sparsamente punteggiato-scabridulo od anche quasi liscio; rachide subtrigono. Segmenti verdi o subconcolori sulle due faccie, più o meno sparsi in gioventù di pagliette forforaceo-ferruginose fugaci nella pagina inferiore, quasi sempre solitarî sopra ogni lato, alterni od opposti con quelli dell'altro lato, raramente vi sono in basso, sopra ognuno dei lati, due segmenti ravvicinati per le basi; se vi è un solo segmento questo ha l'apparenza di esser composto di due ineguali uniti insieme; in generale $i$ segmenti sono romboidali o trapezoidali, lunghi 10-12 cm. e larghi $4-5 \mathrm{~cm}$; cuneati in basso, e da circa la metà in su triangolari a contorno ineguale, acutamente denticolato-premorso: sono poi bruscamente contratti nel mezzo all' apice in una punta acuminata, angusta, spesso caudiforme e lunga 15-20 mm.; il segmento centrale è più grande dei laterali, \pm cuneato-flabelliforme, 3-5-costulato, 
spesso asimmetrico, a contorno con tanti lobi acuminati, o più o meno caudati, quante sono le costole.

Spadici al solito modo monoici (coi fiori maschili molto più precoci dei feminei) eretti od eretto-nutanti, piuttosto brevi, terminali e laterali, solitarî, indivisi, consistenti in una sola spiga fiorifera lunga $6-10 \mathrm{~cm}$., portata da una parte pedunculare lunga poco più o poco meno quanto la spiga, e vaginata quasi per intero da varie spate. Le spate sono più o meno distanziate fra di loro, fortemente striate all'esterno, le più basse brevi e quasi squamiformi, le seguenti brevemente tubulose in basso, aperte su di un lato; esse si prolungano in un lembo non strettamente guainante, allungato-auriculare, acuto; la parte dello spadice rivestita dalle spate $\dot{e}$ di $4-5 \mathrm{~mm}$. di diam. ed il nudo asse di $1.5-3 \mathrm{~mm}$.

Fiori maschili al solito modo molto più precoci dei feminei, obovati, rotondati in alto, attenuati assai in basso, lunghi $5 \mathrm{~mm}$. e larghi 3; calice cupolare, troncato; sepali suborbicolari poco più larghi che lunghi, a contorno rotondato, intiero e finamente ciliolato, carnoso-gibbosi o subcalcarati alla base; corolla 3 rolte più lunga del calice, divisa sino in basso in 3 filli navicolari, subspatolati, coll'apice largo ma acuto, attenuati in basso, con la base acuta, fortemente striati all'esterno; stami circa 30 ; antere lineari, angustissime, ottusiuscule alle due estremità, a loggie parallele; filamenti riuniti insieme alla base, tenuissimi, più corti delle antere. Rudimento d'ovario 0 .

Fiori feminei sviluppantesi al solito modo assai dopo quelli maschili, orizzontali ed inseriti spiralmente ed assai regolarmente intorno l'asse che ricuoprono piuttosto densamente: sono globosi, di circa $5 \mathrm{~mm}$. di diam.; brattee florali reniformi, sepaloidee, fortemente striate; calice spianato a sepali suborbicolari ma un poco più larghi che lunghi, a contorno rotondato, finamente ciliolato, fortemente striato-nervosi; corolla due volte più lunga del calice, divisa sino quasi alla base in 3 segmenti concavi, larghi, subdeltoidei, ottusiusculi ed abbraccianti l'ovario, fortemente 
striato-nervosi. Staminodi O. Ovario globoso, leggermente depresso, trilnculare, rotondato in alto ed ivi con l'accenno di 3 superficialissime carene radianti dagli stigmi; questi sessili, trigoni, conniventi, formanti colla loro riunione una piccola areola circolare nera, piana.

Frutti sferici, quando perfettamente maturi di circa $12 \mathrm{~mm}$. di diam., a pericarpio leggermente carnoso, segnati dalla piccola areola rotonda piana ed immutata sul vertice e con appena un accenno di 3 carene radianti da quella. Semi 2-3, obovati, rotondati in alto, attenuati alquanto verso una base acuta, a dorso convesso e con due faccette separate da un angolo ottuso dal lato interno, lunghi 8-9 e larghi $7 \mathrm{~mm}$, quasi neri, opachi; embrione situato verso la metà del lato dorsale.

Habitat. - Nel Tonkino occidentale: Père Bon, n. ${ }^{\circ} 2382$ e n. ${ }^{\circ} 2528$ a Kien-khè $\theta$ n. ${ }^{\circ} 4646$ a Vo-xa. Presso ThauMoï nelle foreste. della catena calcare: Balansa n. ${ }^{\circ} 518$; e nelle foreste delle vicinanze di Phuong-Lam, Balansa n. ${ }^{\circ}$ 4364. Esemplari tutti nell'Erbario di Parigi.

Osservazioni. - Sono da prima stato tentato di considerare come specie distinta dalla $D$. caudata questa forma, che si distingue dalla tipica per le sue dimensioni minori, per $i$ segmenti delle foglie più piccoli ed in proporzione della lunghezza loro più larghi; per gli spadici più corti, e sopratutto per $i$ fiori maschili clavati e rotondati in alto. $\mathrm{Ma}$ il $\mathrm{n} .^{\circ} 92$ di Spire del Laos, che ho riportato alla forma tipica, viene a togliere lo stacco fra la Didymosperma del Tonkino e quella della Cocincina meridionale, in modo che non mi è sembrata opportuna una distinzione specifica fra le due. La $D$. caudata v. tonkinensis si avvicina molto alla D. nana Griff. dell'Assam, dalla quale differisce principalmente per l'asse dello spadice che è assai densamente forforaceo-rubiginosa nella $D$. nana, ed è glabra in tutte le forme della $D$. caudata. Le spate della $D$. nana sono anche leggermente inflate e non come quelle della $D$. caudata 
strettamente guainanti. Nelle dimensioni però e nella forma delle foglie la $D$. caudata tonkinensis rassomiglia moltissimo alla D. nana.

\section{Wallichia gracilis Becc. sp. $\mathrm{n}$.}

Coespitosa, caudicibus fertilibus circ. $1.5 \mathrm{~m}$. altis, dum vaginis foliorum indutis $2-2.5 \mathrm{~cm}$. crassis; foliis radicalibus longe, superioribus breviuscule, petiolatis, petiolo tereti, 8-10 mm. diam.; segmentis elongato-cuneatis, basilaribus $30-40 \mathrm{~cm}$. longis, superne $8-9 \mathrm{~cm}$. latis, papyraceis, viridibus, subtus pallidioribus et in juventute subalbicantibus, minute fusco-punctulatis, margine anteriore undulato inaequali et denticulato-serrato. Spadices terminales et laterales, dioici, $30-35 \mathrm{~cm}$. longi, recurvi, spatis auriculaeformibus, numerosis, furfuraceo-tabacinis, indutis. Spadicis masculi panicula anguste oblonga, ramulis numerosis gracilibus; floribus masculis ovatis, obtusis, $5 \mathrm{~mm}$. longis, sessilibus; calyce brevissimo; corollae phyllis oblongo-navicularibus. Spadicis foominei panicula fructifera ovata, densa, ramulis brevibus, 5-6 $\mathrm{mm}$. longis; fructibus ovatoellipticis, utrinque aequaliter rotundatis, $13 \mathrm{~mm}$. longis, $8 \mathrm{~mm}$. crassis, apice mammillato; perianthio fructifero de. presso-cupulari, $7 \mathrm{~mm}$. diametro.

Descrizione. - Cespitosa; i germogli non ancora fioriferi sono acauli; quelli fertili sono gracili e fogliuti, alti $1.5 \mathrm{~m}$. e muojono dopo la maturazione dei frutti (Balansa). Il fusto rivestito dalle guaine delle foglie è apparentemente di soli $2-2.5 \mathrm{~cm}$. di diam.

Foglie radicali e della parte bassa dei fusti molto lungamente picciolate (non viste intiere); in una il picciolo $\dot{\theta}$ lungo $90 \mathrm{~cm}$., terete, di 8-10 mm. di diam.; nelle fronde giovani il picciolo è intieramente coperto da un indumento forforaceo-rubiginoso, ma poi diventa glabrescente e molto finamente scabridulo. Segmenti cartacei, verdi di sopra, più 
pallidi e nelle fronde giovani subalbescenti di sotto e quivi distintamente sparsi di minutissimi puntolini scuri; essi hanno la costola mediana sottile, tondeggiante di sotto, e quivi più rilevata che di sopra; i nervi secondarî sono numerosi, poco più forti dei terziarii ; questi sono numerosissimi, tutti molto sottili ma rilevati, e rendono distintamente radiatostriate le due superficî; i segmenti inferiori delle fronde giovani sono lungamente cuneati verso una base molto acuta, hanno il margine apicale od anteriore asimmetrico ed ondulato, $\theta$ sone \pm prolungati all'apice in punta irregolarmente triangolare acuta od ottusiuscula: sono lunghi $30-40 \mathrm{~cm}$. e larghi $8-9 \mathrm{~cm}$. verso la metà. Le foglie della parte apicale e fiorifera del fusto sono assai più piccole delle radicali, hanno il picciolo molto più corto, la parte pinnifera (in un esemplare) $\dot{\theta}$ lunga $50 \mathrm{~cm}$. ed ha 6 segmenti per parte oltre quello terminale; tali fronde hanno la vagina con una spessa costola dorsale $\theta$ nel rimanente sfilaccicato-reticolata con fibre nere molto sottili; la ligula (nelle fronde giovani) è lunghissima (20-30 cm.), acuminata, rubiginosoforforacea all'esterno, presto lacera $\theta$ ridotta in fibre parallele, sottilissime, nere; il rachide è rubiginoso-forforaceo ed è bifaciale con angolo non molto acuto di sopra; i segmenti della parte superiore del rachide sono orizzontali, cuneati in basso, a margini inferiori o laterali piuttosto corti, la punta loro è subtriangolare ottusa, sono lunghi 18-20 cm., larghi 5-6 cm.; il segmento terminale è flabelliforme con 3-4 lobi superficiali, denticolati, ottusi.

Spadici pochi, spesso solitarii e terminali od accompagnati da pochissimi altri laterali; apparentemente dioici, però $i$ maschi ed i feminei sono pochissimo differenti fra di loro e sono costituiti da una pannocchia lunga $15-18 \mathrm{~cm}$., portata da una parte peduncolare un poco più lunga della parte fiorifera, terete, di 6-7 mm. di diametro e che subito sin dal momento dell'antesi è fortemente arcuata, e porta la pannocchia rivolta in basso; la parte peduncolare è avvolta da 6-7 spate relativamente grandi, imbricate, mollemente forforaceo-rubiginoso-tabacine all'esterno, glabre e 
finamente striate internamente, sottilmente coriacee, lanceolato-acuminate, concave ed in forma d'orecchio d'asino, non tubolose e non abbraccianti in basso, le più esterne brevi, le interne gradatamente più grandi, le maggiori lunghe 12$15 \mathrm{~cm}$. Pannocchia dei fiori $\sigma^{\prime}$ densa, molto strettamente oblunga, un poco attenuata all'apice, composta di numerosissimi ramoscelli inseriti spiralmente intorno all'asse, paten. tissimi, arcuato-ascendenti, sottili (di $1 \mathrm{~mm}$. di spessore), i più bassi lunghi circa $2 \mathrm{~cm}$.

Fiori maschili ovati, ottusi, lunghi $5 \mathrm{~mm}$., inseriti spiralmente intorno ai ramoscelli, sessili all'ascella di una minutissima bratteola membranacea, concava, acuta; calice brevissimo; segmenti della corolla oblungo-naviculari, fortemente striati.

Pannocchia fruttifera ovata, molto densa, con ramoscelli irregolarmente inseriti a spirale, ascendenti (o penduli poichè l'intiera pannocchia è reflessa), glabri, lunghi 5-6 cm., spessi $2-3 \mathrm{~mm}$; fiori 우 inseriti alquanto irregolarmente a spirale, accolti framezzo a 2 piccole brattee formanti un culiculo, concave, semiorbicolari a contorno rotondato.

Perianzio fruttifero apparentemente non accresciuto, depresso-cupolare, accogliente tutta la base del frutto, di $7 \mathrm{~mm}$. di diam.; calice spianato a contorno 3-lobo, lobi rotondati, striati; corolla 2 volte più lunga del calice, divisa sino circa alla metà in 3 larghi lobi deltoidei, crassi, ottusiusculi.

Frutto ovato-ellittico, egualmente rotondato alle due estremità, mammillato all'apice o marcato da una piccola areola circolare di $2 \mathrm{~mm}$. di diametro. I frutti immaturi sono lunghi $13 \mathrm{~mm}$. e larghi 8. Seme....

Habitat. - Nel Tonkino a Dong-Dang, nelle foreste, Balansa n. ${ }^{\circ} 519$ (Febbr. 1885) e n. 4369 (esemplare con frutti immaturi (19 Genn. 1886), nelle foreste del Monte Bavi a circa $800 \mathrm{~m}$. d'altezza, e n. 4362 (27 Decembre 1887) con la nota: "Dioique? Tronc débile de m. 1,50 de hau- 
teur. Vallée de Banton, dans les bois ", esemplare con fiori maschi. (Erb. di Parigi).

Osservazioni. - Sembra la specie più piccola del genere. Distinta fra tutte per i suoi gracili fusti portanti in alto o lateralmente dei relativamente piccoli spadici ; per la pannocchia maschile angusta con corti ramoscelli fioriferi $e$ piccoli fiori; per la pannocchia fruttifera ovata con ramoscelli corti; per i segmenti verdi sulle 2 faccie e fugacemente albicanti di sotto, similissimi del resto a quelli della W. caryotoides.

Alla $W$. gracilis sembrano riferibili gli esemplari raccolti nell'Yunnan da Henry, $\mathrm{n}^{\circ} 12331$ (sole fronde), delle montagne Szemao, ed il n. 10411 con frutti immaturi raccolti sulle Montagne Menghi a 500 piedi di altezza. (Vidi nell'Erb. di Calcutta).

\section{Iicuala (Iicualella) tonkinensis Becc. sp. n.}

Gracilis. Frondium lamina dimidiato-orbicularis, radiatopartita; segmentis 6-8 inaequalibus, omnibus apice obtuse dentatis; lateralibus elongato-cuneatis, 5-6-costulatis, extimis oblique truncatis, segmento centrali lateralibus conspicue latiori, basi non pedicellato, in medio profunde inciso, divisionibus 8-10-costulatis; petiolo in parte basilari tantum spinis parvis conicis horizontalibus armato. Spadix (in specimine uno circiter $5 \overline{5} \mathrm{~cm}$. longus) strictus, rigidulus, undique indumento tenui rubiginoso-furfuraceo obtectus, indivisus, parte axili spathis duabus completis vaginata, crassiuscule filiformi, basi compressiuscula, $4 \mathrm{~mm}$. lata, superne subtereti et $3 \mathrm{~mm}$. diametro, sensim in partem florigenam minime incrassatam, circ. $10 \mathrm{~cm}$. longam, continuata; spathis minute rubiginose furfuraceis, membranaceis, exsuccis, basi arcte vaginantibus, superne nonnihil inflatis, valde compressis, apice tantum perviis et laceromarcescentibus. Flores ovati, $4.5 \mathrm{~mm}$. longi, horizonthales, 
sessiles, circum spicam spiraliter dispositi, pulvinulo superficiali insidentes; calyce late-campanulato, basi planiuscula callosa, limbo membranaceo extus rubiginose papilloso-furfuraceo, irregulariter 3-lobo ; corolla calyce subduplo longiori, segmentis elongato-triangularibus, subtiliter coriaceis, extus glabris, striato-venosis; ovario glabro; carpellis abortivis basilaribus.

Descrizione. - Gracile. Fronde (due sole esaminate) con picciolo lungo 40-45 cent., della uniforme larghezza di $4 \mathrm{~mm}$., triangolare da cima a fondo, piano di sopra, con lo spigolo inferiore ottuso, ed i margini acuti armati solo presso la base con poche spine, piccole, coniche, orizzontali; ligula apicale cordata, callosa; lembo nell' insieme semiorbicolare, palmato, diviso sino alla base in 8 segmenti ineguali; il mediano latamente $\theta$ sin dall'apice gradatamente cuneato, non pedicellato alla base diviso nel mezzo per i $2 / 3$ della sua lunghezza; ogni divisione ha 8-10 nervi primarii; $i$ segmenti laterali sono più stretti ed hanno 5-6 nervi; il segmento più esterno è il più angusto, tutti sono cuneati, ma i più esterni sono leggermente ristretti in alto, e troncati obliquamente, tutti sono terminati da denti brevi, separati da seni ottusi, ogni dente è brevemente bilobo all'apice con i lobi ottusissimi.

Spadici dirittissimi, indivisi, minutamente rubiginosoforforacei in ogni parte, con la porzione peduncolare lunga 45 cent., intieramente guainata da due spate, compressa alquanto e lurga dalla base sin presso l'apice $4 \mathrm{~mm}$.: quivi è cilindracea, di $3 \mathrm{~mm}$. di diametro, e gradatamente senza demarcazione passa nella spiga fiorifera. Spate due, finamente rubiginoso-forforacee, quasi eguali di forma, l'interna però circa $\mathrm{di}^{1} / 3$ più lunga dell'esterna, membranacee, essucche, lungamente tubulose, strettamen te guainanti in basso, leggermente inflate nella parte superiore, fortemente compresso-ancipiti, coi lati molto acuti, aperte solo all'apice ed ivi bifide $\theta$ terminate in punta acuta, \pm marcescente e sfilaccicata. La spiga o parte fiorifera è terete ed allun- 
gata e porta i fiori a spirale tutto ingiro sopra pulvinuli superficiali, non sporgenti.

Fiori solitarî, sessili, lunghi $4.5 \mathrm{~mm}$. ; calice campanulato, calloso in basso, a lembo membranaceo diviso in 3 lobi triangolari acuti, densaniente forforaceo-papillosi, rubiginoso di fuori; corolla un poco meno del doppio più lunga del calice, sottilmente coriacea, a lobi triangolari allungati striati all'esterno. Giovani frutti ovoidei-oblunghi, glabri.

Habitat. - Scoperta dal Padre Bon nel Tonkino occidentale a Vo-xa nelle selve rupestri del monte Chuà Hac, il 5 maggio 1885, n. 2901. Nome volgare «Luà Khua ». (Erb. De Candolle e di Parigi).

Osservazioni. - $\dot{\mathrm{E}}$ molto ben caratterizzata per lo spadice indiviso con parte fiorifera non incrassata. Si avvicina alla Licuala (Licualella) mattanensis Becc., dalla quale differisce per i segmenti larghi, di cui il mediano profondamente bipartito $e$ per i frutti ovato-oblunghi. Ho visto solo una fronda ed uno spadice con frutti immaturi. La specie però è ben caratterizzata.

\section{Iicuala calciphila Becc. sp. n.}

Parvula, gracillima. Frondes inter minores, lamina parva, 3-5-partita, segmento centrali latissime cuneiformi, 15-16 $\mathrm{cm}$. longo, usque ad medium et ultra in medio fisso, basi non pedicellato, divisionibus 5-7-costulatis apice truncatis et obtuse dentatis; segmentis lateralibus linearibus vel anguste cuneatis et jam ab. apice, oblique et argute dentato, basin versus sensim attenuatis; petiolo gracillimo, 20-30 cm. longo, $2 \mathrm{~mm}$. lato, spinis rectis validiusculis, 2-4 mm. longis, basi subbulbosis, ad margines armato. Spadices graciles, arcuato-nutantes, petiolis breviores, in ramos 2-3 remotiuscule superpositos divisi; Spathis primariis tenuiter membranaceis, marcescentibus, 
tubulosis, basi lassiuscule vaginantibus, superne anguste auriculatis, acuminatis; ramis in 2-3 ramulos florifer os graciles, 4-5 $\mathrm{cm}$. longos, vix $1 \mathrm{~mm}$. crassos, divisis; floribus parvis spiraliter insertis, plane sessilibus, ovatis, acutis.

Drscrizione. - Gracilissima, stolonifera, con fusti gracili di $60 \mathrm{~cm}$. di altezza (Balansa), foliosi all'apice, di $7 \mathrm{~mm}$. di diam. in basso nella parte nuda.

Foglie fra le più piccole; guaina intiera, sottilmente coriacea, carinata sul dorso e quasi iutiera, lunga circa $5 \mathrm{~cm}$., brevemente tubulare in basso, sottilmente coriacea e carenata sul dorso, fibroso-reticolata sul lato ventrale, specialmente in alto, dove si dilata alquanto $\theta$ dove si prolunga anteriormente in una ocrea liguliforme, lunga $5-6 \mathrm{~cm}$., membranacea, essucca, presto marcescente e che si sfacela in filamenti sottili e flaccidi; picciolo gracilissimo, lungo 20-30 cm., largo $2 \mathrm{~mm}$, subtrigono, piano e striato di sopra, con angolo assai prominente di sotto, con margini acuti armati di spine orizzontali relativamente assai forti, ma non molto titte, dritte, lunghe $2-4 \mathrm{~mm}$, con base inspessita e quasi bulbiforme; ligula triangolare-allungata; lembo assai più corto del picciolo, sottile cartaceo, opaco sopra ambedue le faccie, leggermente più pallido di sotto, diviso in 3-5 segmenti, dei quali il centrale partito in due sino alla metá o sino al terzo inferiore, nell' insieme molto latamente cuneiforme $\Theta$ largo in alto $8-10 \mathrm{~cm}$., non pedicellato alla base, lungo 15-16 cm.; ogni sur divisione ha 5-7 costole primarie superiori ed all'apice $\dot{\theta}$ troncata con i denti primarii larghi e bassi ed i secondarii ottusi ; se i segmenti sono cinque, i 2 più esterni sono molto piccoli e lineari; gli intermedî hanno 3-5 costole primarie : sono gradatamente cuneati sin dall'apice che è obliquo e più profondamente dentato che nel segmento centrale.

Spadici arcuato-nutanti dalla ascella delle foglie e più corti dei piccioli di queste, gracili, con 2-3 palchi di rami sovrapposti; i rami sono divisi in 2-3 ramoscelli fioriferi; spate primarie sottili, membranacee, marcescenti, guainanti 
completamente l'asse dello spadice, tubulose, lassamente guainanti in basso, prolungate in lunga punta strettamente auriculare, acuminata; ramoscelli foriferi gracili, lunghi 4-5 cm., e di appena $1 \mathrm{~mm}$. di spessore, portanti i fiori non molto fitti ed a spirale.

Fiori piccoli, affatto sessili, ovati, acuti (visti solo in cattivo stato).

Habitat. - Tonkino: sulle rupi calcaree in vicinanza della sponda sinistra del Fiume nero a 3 chilometri a monte di Phuong-Lam, nella foresta d'alto fusto, raccolta da B. Balansa il 2 maggio 1888. (Erb. di Parigi).

Osservazioni. - 亡̀ una delle più piccole specie, e che per le foglie rassomiglia alla L. ternata. É distinta per i suoi piccoli spadici con 2 o 3 soli palchi di rami, pochissimo divisi, più corti dei piccioli e vaginati completamente da varie spate membranacee, lassamente guainanti, marcescenti, terminate in stretto e lungo lembo acuminato auriculare, e per i piccoli fiori sessili.

Iicuala fatua Becc. sp. n.

Gracilis. Folia.... Spadices elongati, graciles, rigidi, recti, in 3-4 ramos primarios remote superpositos divisi; parte axili tenui, circiter $2 \mathrm{~mm}$. diametro, spathis tubulosis, compressis, exsucce membranaceis, omnino vaginata; ramis in 4-5 ramulos floriferos irregulariter partitis; ramulis floriferis 5-15 mm. longis, gracilibus, sinuosis, subulatis. Flores spiraliter secus ramulos inserti, plane sessiles, ovati, basi rotundati, superne paullo attenuati, 3-3.5 mm. longi, $2 \mathrm{~mm}$. crassi ; calyce cyathiformi, membranaceo, profunde 3-dentato, puberulo; corolla calyce $1 / 3$ longiori, usque ad medium in 3 segmenta triangularia pergamenacea extus striato-venosa partita; staminibus infra medium corollae insertis, flamentis e basi crassa teretibus subulatis, antheris 
suborbicularibus, parvis, filamentis triplo vel quadruplo brevioribus ; carpellis glabris; subclavatis et in stylum conicum subulatum superne attenuatis.

Descrizione. - Pianta apparentemente assai gracile. Foglie... Spadici allungati, gracili ma rigidi e dritti, con 3-4 (o forse anche più) palchi di rami sovrapposti; la parte assile è molto sottile, di $2 \mathrm{~mm}$. di diametro ed é intieramente inguainata dalle spate; i rami escono eretti dalla bocca delle respettive spate e si dividono irregolarmente in pochi (4-5) ramoscelli fioriferi (non sono quindi digitati); spate primarie allungate, tubulari, compresse, molto lassamente guainanti, sottili, membranacee, essucche, brune, parzialmente squamuloso-forforacee, lacero-fesse in alto; l' inferiore è ancipite e lunga circa $20 \mathrm{~cm}$. e larga $15 \mathrm{~mm}$. in alto, ed è ristretta in basso; ramoscelli fioriferi lunghi 5-15 cm., gracili, sinuosi, spessi $1.5 \mathrm{~mm}$. alla base, subulati all'apice, forforacei o rivestiti assai densamente da peli ramentacei rubiginosi; i ramoscelli portano tutto in giro i fiori, che riposano sopra tubercoletti superficialissimi; bratteole florali inconspicue.

Fiori affatto sessili, ovati, spesso alquanto asimmetrici, rotondati in basso, attenuati verso l'apice, ma ottusiusculi, lunghi $3-3.5 \mathrm{~mm}$., larghi circa $2 \mathrm{~mm}$.; calice ciatiforme, membranaceo, fortemente striato-nervoso, assai profondamente 3-dentato, con i denti triangolari; questi sono ciliati sul margine, e specialmente all' apice, con peli ramentacei ferruginei; simili peli sono sparsi anche sul corpo del calice, specialmente alla base; corolla un terzo più lunga del calice, attenuata in basso, divisa sino circa la metá in 3 segmenti triangolari, allungati, pergamenacei, striati esternamente; stami inseriti poco al di sotto della metà della corolla, tutti eguali, con filamenti tereti, allungati, 3-4 volte più lunghi delle antere, dilatati e con un ringrosso bulbiforme in basso, dove con le loro basi a contatto l'una con l'altra formano, alla fauce della corolla, un anello carnoso 6-lobo; antere piccole suborbicolari; carpelle glabre, 
allungate, subclavate, assai attenuate in basso, non scolpite in alto ma spesso con depressioni sui lati causate dalla pressione della base dei filamenti, ristrette nel terzo superiore in uno stilo subulato a base conica; stigma punti. forme. Frutti mancano.

Habitat. - Nel Tonkino occidentale. Père Bon, n. ${ }^{\circ} 3130$ nell' Erbario di Parigi.

Osservazioni. - Di questa specie ho visto un solo spadice mutilato dell'estremità ; la porzione assile esistente $\dot{e}$ lunga $35 \mathrm{~cm}$. e porta 2 rami lunghi $18-20 \mathrm{~cm}$.; $\dot{\theta}$ guainata dalla spata basilare e da altre 2 spate, una per ognuno dei rumi. Lo spadice intiero lo giudico di circa $60 \mathrm{~cm}$. di lunghezza. Mancano le foglie. Ad onta dell' incompleto materiale apparisce una delle specie meglio caratterizzate per i suoi niccoli fiori ovati, sessili, con calice peloso-ramentaceo, ma sopratutto per gli stami con filamenti relativamente molto allungati $\Theta$ robusti, che rassomigliano quelli delle specie della sezione Dammera, mentre in generale in tutte le Eulicuala i flamenti degli stami sono minutissimi $\theta$ dentiformi.

Rhapis micrantha Becc. sp. n.

Gracilis, 1-2 m. alta, caudice nudo $8 \mathrm{~mm}$., dum vaginis induto $15-20 \mathrm{~mm}$. diametro. Frondium vagina et ligula concinne in fibras solutae, fibris exterioribus complanatis, interioribus tenuioribus, criniformibus, teretibus ; segmenta 6-10 usque ad basin omnino libera. Flores masculi turbinati, 4-4.5 mm. longi; calyce membranaceo 3-lobo, lobis acutis; corolla calycem triplo superanti; staminum flamentis corollae adnatis, usque ad apicem linearibus; antheris paullo latioribus quam longioribns. Flores foeminei clavati, dum bene evoluti $4.5 \mathrm{~mm}$. longi; corolla superne subglobosa, basi longe angusteque attenuata; calyce basi carnoso, 3- 
dentato, dentibus triangularibns acutis. Fructus albi, sao. pius 3-ni, globosi, 8-9 mm. diam., semine globoso, 6-6.5 $\mathrm{mm}$. diametro.

Descrizione. - Dioica (?); molto gracile, alta 1-2 m. Tronco vaginato di $15-20 \mathrm{~mm}$. di diametro; il tronco nudo di soli circa $8 \mathrm{~mm}$. Vagine tubolose, leggermente dilatate in alto, lunghe circa $20 \mathrm{~cm}$., ottusamente carenate e coriaceo-legnose lungo quasi tutto il lato dorsale, risolute sul lato ventrale in un reticolo formato da 2 strati di fibre di color bruno-castagno; nello strato esterno le fibre sono ascendenti obliquamente, filiformi ma alquanto schiacciate e larghe spesso sino quasi $1 \mathrm{~mm}$., disgregate l'una dall'altra e riapprossimate lungo la linea mediana dal lato ventrale, dove quelle della parte più alta concorrono a formare una ligula triangolare, lunga sino a $3 \mathrm{~cm}$., opposta al picciolo e non sfacelata; le fibre dello strato interno della vagina incrociano quelle più esterne seguendo una direzione longitudinale e sono di queste molto più sottili; tanto le une quanto le altre si discostano alquanto l'una dall'altra ma rimangono nella posizione loro e nell'insieme formano un lasso reticolo, non s'increspano e non si arruffano come più o meno accade nella Rh. fabelliformis. Picciolo lungo 20-30 cm., gracile, biconvesso o piano-convesso, almeno in basso, con i margini scabriusculi o quasi lisci, piuttosto acuti, specialmente verso l'alto; nelle fronde giovani è barbato-lanoso sul contorno. Lamina delle foglie radiato-digitata, nell'insieme con circa 20 costole primarie superiori, divisa in 6-10 segmenti cartacei, verdi e non nitenti di sopra (sul secco), a mala pena più pallidi di sotto, ineguali, lunghi $20-25 \mathrm{~cm}$., 1-5-costulati, larghi $0.5-$ $4 \mathrm{~cm}$., espansi in semicerchio, tutti liberi sino in basso; i segmenti centrali sono spesso i più larghi, tutti si terminano all'apice in 2-5 denti triangolari, acuminati e brevemente bifidi; i segmenti laterali hanno i denti più stretti e più acuminati dei centrali; le costole (o nervi primarî) superiori ed inferiori ed i margini sono minutamente $\theta$ poco 
argutamente serrulato-scabriduli; venule transverse numerose, acute.

Spadici \pm densamente coperti nelle parti assili, all'epoca della fioritura, da un indumento forforaceo-ramentaceo, ferrugineo, poi glabri. La parte peduncolare dello spadice $\dot{\theta}$ completamente vaginata da due spate generali ed è quasi del tutto inclusa nella guaina della foglia all'ascella della quale esce; essa porta solo 3-4 piccole infiorazioni parziali formanti una breve, lassissima e diffusa pannocchia. Le spate primarie sono membranacee, striate per il lungo, tubulose, aperte all'apice, dove si termisano in una punta lanceolata, fibroso-lacera e piú o meno coperta da un indumento ramentaceo-forforaceo; la spata più bassa è lunga circa $15 \mathrm{~cm}$, acutamente bicarinata; la seconda spata sorpassa alquanto l'esterna; la parte assile che rimane fuori della spata è sinuosa a zig-zag, con lo spazio fra un ramo e l'altro dritto e vaginato da una spata tubulosa più lunga del ramo stesso, aperta da un lato dalla metà in su, e terminata in punta acuminata che abbraccia la base dei rami primarii; tutte queste spate sono membranacee $\theta \pm$ forforaceo-ramentacee all'esterno. I rami primarî sono patentissimi, e come l'apice dello spadice sono composti di varî ramoscelli fioriferi, sparsi, patenti, gracilissimi, filiformi, subulati, lunghi $4-8 \mathrm{~cm}$. (i più corti essendo i più alti) ed aventi i pulvinuli dei fiori superficiali.

Fiori maschili turbinati, quando completamente sviluppati, lunghi 4-4.5 mm., disposti, senza molta regolarita a spirale, sessili, solitarî, orizzontali, muniti in basso di una minutissima bratteola jalina, acuminata, decidua. Calice membranaceo, cupolare-campanulato, subjalino, molto minutamente punteggiato-glanduloso, profondamente 3-lobato, coi lobi triangolari e non di rado lobulati alla lor volta, acuti od anche apiculato-caudati. Corolla turbinata, carnosula, attenuata in basso, al momento dell'antesi due volte più lunga del calice, brevemente 3-loba all'apice, coi lobi rotondati a mala pena apicolati e muniti internamente, in punta, di una callosità crestiforme sulla linea mediana che 
separa due incavi o nicchiette, in ognuna delle quali si annida la metà di una antera. Stami 6 , subiseriati, i tre corrispondenti ai seni fra un lobo e l'altro essendo un poco più lunghi degli altri tre; $i$ filamenti sono tereti e si partono quasi dal fondo della corolla, ma sono connessi a questa per tutta la lunghezza loro; antere suborbiculari con largo connettivo, a loggie subreniformi deiscenti sui lati. Rudimento d'ovario inconspicuo o piccolissimo, oblungo, carnosulo.

Spadici feminei simili a quelli maschili, ma con minor numero di fiori, e questi più radi sui ramoscelli. I fiori feminei sembrano a prima vista di due qualita, alcuni essendo globosi ed altri clavati e più lunghi dei maschili; ma forse al momento dell'antesi anche ai feminei si allunga il tubo della corolla. I fiori globosi sono di circa $2 \mathrm{~mm}$. di diam.; il calice è membranaceo, cupolare, irregolarmente trilobo, a lobi ottusamente denticolati ed apicolati. Corolla divisa sino alla metà in 3 lobi quasi valvati, latamente ovati, apicolati, concavi, molto crassi. Stami sterili 6 , con filamento quasi nullo; le antere sono vacue, ma ben conformate e grandi, cordato-ovate, smarginate all'apice con largo connettivo, con le linee della deiscenza laterali. Ovario composto di 3 carpelle libere fra di loro e nell'insieme latamente turbinato; carpelle convesse dal lato esterno, bifaciali dal lato interno, bruscamente contratte in breve stilo con stigma puntiforme; ovulo inserito in basso della loggia, eretto. Alcuni esemplari hanno fiori che differiscono esternamente da quelli ora descritti, perchè, a quanto sembra, sono completamente sviluppati e si trovano al momento dell'antesi; questi fiori sono allora clavato-capitellati od in forma di chiodo di garofano; la corolla loro ha una capocchia quasi globosa di $1.7 \mathrm{~mm}$. di diam., bruscamente attenuata in basso in una parte piuttosto sottile, piena. Nell'insieme la corolla è allora $2 \frac{1}{2}$ volte più lunga del calice; le carpelle rimangono in alto nella parte dilatata della corolla, sorrette da una specie di colonna costituita dalla parte assottigliata e piena della corolla; esse sono esattamente 
come di già state descritte e circondate dagli usuali stami sterili.

Frutti globosi, spesso 3-ni, risultanti dallo sviluppo indipendente delle 3 carpelle, bianchi sul vivo (Bon), di $8-9 \mathrm{~mm}$. di diam. sul secco: in questo stato sono opachi $\theta$ di un color scuro all'esterno; epicarpio sottile, unito; mesocarpio allo stato secco granuloso-spongioso, ma probabilmente leggermente carnoso sul fresco, staccantesi facilmente a completa maturitá dal nocciolo (endocarpio); l'endocarpio $\dot{e}$ sottile, fragile, crostaceo-legnoso. Il seme si libera con difficoltà dall'endocarpio, è globoso, di 6-6.5 mm. di diametro, un poco pianeggiante dal lato dell' intrusione del rafe; il rafe è assai largo, leggermente impresso, gira intorno alla meta inferiore del seme e dall'altro lato termina all'embrione, che si trova nel centro della faccia più convessa; il processo del rafe forma una massa spongiosa, globosa, scura, che penetra sino alla metà del seme; l'albume è osseo ed in sezione longitudinale si presenta della forma di ferro di cavallo; la testa del seme è opaca, bruna, senza ramificazioni del rafe distinte. Il perianzio fruttifero forma un distinto pedicello slargato in alto e che accoglie le tre drupe.

Habitat. - Nel Tonkino occidentale sui monti DôngBaù a Kien Khê. Scoperta dal Padre Bon, 19 Aprile 1884. Nome volgare « Kay Lui ». N. ${ }^{\circ} 2545$, esemplari con fiori đ nell'Erb. De Candolle e di Parigi e n. ${ }^{\circ} 2546$ nell' Erb. De Cand.; $n .^{\circ} 2345$ esemplare in frutto e con fiori $q$ (clavati), nell'Erb. di Parigi.

Osservazioni. - Ha intieramenta l'abito della Rh. fabelliformis, dalla quale si distingue principalmente per la guaina delle foglie, la quale è composta nella sua parte ventrale di fibre che si discostano l'una dall'altra, ma che rimangono al loro posto $\theta$ non si arruffano; di più le fibre esterne sono complanate e più grosse delle interne che per di più sono tereti; si distingue inoltre per la ligula non sfa- 
celata; per i segmenti completamente liberi fra di loro sino in basso; per gli spadici più allungati e per i fiori maschili con calice a lobi ineguali, spesso apicolati, e per i petali più rotondati in alto; infine per i fiori feminei più piccoli.

E anche affine alla $R h$. humilis per le sue guaine a fibre non arruffate, ma in questa i segmenti non sono completamente liberi sino in basso.

Rhapis laosensis Becc. sp. n.

Gracilis, caudice nudo $5-7 \mathrm{~mm}$., dum vaginis induto 10-15 mm. diam. Frondium vagina et ligula in fibras tenuissimas molles, concinnas, solutae; segmenta saepe tantum 3 et pluricostulata, interdum usque ad 9 et 2-3-costulata, omnia, vel saltem centralia, basi plus minusve connata. Spadices spathis auriculiformibus subduplo longiores. Flores masculi $4 \mathrm{~mm}$. longi; corolla clavata, basi valde angustata, calyce quadruplo longiori; calyce 3-dentato, dentibus triangularibus acutis; staminibus biseriatis, antheris suborbicularibus; flores foeminei obovati, basi attenuati, $4 \mathrm{~mm}$. longi, $2.8 \mathrm{~mm}$. crassi.

Descrizione. - Gracile. Il tronco allorchè coperto dalle vagine delle foglie è di $10-15 \mathrm{~mm}$. di diam.; il tronco nudo solo di 5-7 mm., con internodi discosti 20-25 mm. nella parte più alta, ed in basso solo 6-7 mm. Vagine lunghe 7-10 cm., nell' insieme cilindraceo-infundibuliformi, ottusamente carenate e coriaceo-sublegnose lungo il lato dorsale, intiere e tubulari in basso, risolute in reticolo fibroso sul lato ventrale; le fibre sono tutte molto sottili, capillari e morbide, quelle dello strato esterno sono leggermente più forti delle sottostanti e molto regolarmente parallele (non arruffate), concorrenti a formare una assai lunga ligula acuminata, risoluta poi, più o meno, in un reticolo molle. Picciolo gracilissimo, lungo 20-35 od anche solo 10-15 cm., largo 2-2.5 mm., più o meno compresso, a mar- 
gini piuttosto acuti, lisci od anche scabriduli. La ligula all' apice del picciolo è triangolare, barbato-lanosa nelle fronde giovani. Lamina radiato-digitata, nell' insieme, di solito, con 16-17 costole primarie superiori, ma talora sino a 23, divisa spesso in 3 soli segmenti ineguali con 5-7 costole primarie superiori, larghi sino $5-6 \mathrm{~cm}$. e lunghi 20-25 cm.; talvolta i segmenti sono sino a nove e più o meno ineguali, 1-5-costulati e larghi in proporzione del numero delle costole: sono uniti in basso per il tratto di 1-3.5 cm.; quando sono molto larghi, sono distintamente concavn-convessi, quasi egualmente attenuati alle due estre. mità, con i denti apicali acuti e bifidi ma cosi conniventi da rendere i segmenti \pm acuminati; di consistenza, i segmenti, sono cartacei, alle volte sono distintamente nitenti di sopra, più pallidi di sotto; le costole superiori ed inferiori sono \pm serrulato-scabridule; i margini sono molto finamente ed acutamente serrulato-denticolati; venule transverse approssimate, sottili e distinte.

Spadici con pochi rami divaricati e divisi in ramoscelli lunghi pochi centimetri e portanti pochi fiori; nell' insieme gli spadici sono assai brevi, più corti dei piccioli ed alle volte di poco più lunghi delle spate. Le spate sono assai variabili di grandezza, assai grandi, le due inferiori tubulose $\theta$ fortemente compresse in basso, auriculeformi in alto, acute od acuminate, più o meno fugacemente forforacee; di solito una terza spata pure assai grande ed auriculeforme si trova alla base delle diramazioni principali.

Fiori maschili piccoli, clavati, angusti, lunghi $4 \mathrm{~mm}$.; calice acutamente 3-dentato; corolla 4 volte più lunga del calice con i 3 lobi deltoidei, apiculati; stami biseriati; antere suborbicolari a loggie reniformi e largo connettivo.

Fiori feminei orizzontali, provvisti alla base di una bratteola membranacea, subulata, decidua, obovati, crassi, attenuati un poco in basso: $\mathrm{i}$ più sviluppati da me visti sono lunghi 4 e larghi $2.8 \mathrm{~mm}$., con la base della corolla crassa. e che non sembra si allunghi molto al momento della antesi, a giudicare dal perianzio fruttifero; calice cupolare, su- 
perficialmente 3-dentuto; carpelle fortemente gibbose in alto, solcate sul dorso in causa dell'impressione lasciata dal filamento degli stami sterili; stilu breve, rimanente più basso della gibbosità della respettiva carpella. Perianzio fruttifero molto brevemente pedicelliforme. Frutti.....

Habitat. - Indo-China, nel Laos meridionale, valle del Mekong a La-Khon, racc. dal D. Thorel, (senza numero), ed a Saraburg (n. ${ }^{0} 3154$, esemplari con fiori $\nearrow^{7}$ ). Pure nel Laos a Xeng-Kouang. (D. Spire, n. ${ }^{0}$ 568). Esemplari tutti dell' Erb. di Parigi.

Osservazioni. - Si distingue dalla $R h$. micrantha specialmente per la natura delle fibre nelle quali si risolve la parte anteriore delle guaine delle foglie; tali fibre nella Rh. micrantha sono rigide ed appiattite, mentre nella Rh. laosensis sono finissime e molli; tanto nell' una quanto nell' altra dette fibre non sono arruffate ed in questo ambedue differiscono dalla $R h$. Alabelliformis. I fiori feminei sono tozzi e crassi e non sembra che la corolla loro si allunghi molto al momento dell'antesi; tali fiori poi sono particolari per le carpelle che, nel boccio, sono fortemente gibbose ed hanno lo stilo molto corto e situato più basso della gobba della respettiva carpella.

Dalla Rh. subtilis differisce per le dimensioni assai maggiori e per le fronde con maggior numero di segmenti.

\section{Rhapis subtilis Becc. sp. n.}

Pusilla, gracillima, 0.50-1 m. alta, caudice basi nudo et crebre annulato, tenuissimo, $3 \mathrm{~mm}$. diam.; frondium vagina in fibras uniformes tenuissimas molles concinnas soluta; segmenta perpauca, bi-3-costulata, vulgo 3, interdum 2-4, usque ad basin omnino libera, $9-10 \mathrm{~cm}$. longa, apice paullo angustata et irregulariter dentata; spadicis parte pedunculari spatharum subtriplo longiori; spathis angustissimis. 
Descrizione. - Gracilissima, alta 0.50-1 m. con tronco sottilissimo di soli $3 \mathrm{~mm}$. di diam., folioso solo in alto, nodoso in basso, con internodi lucidi, variabili da 6-10 a 20-25 mm. di lunghezza. Vagine brevi, disintegrate sul lato ventrale in fibre capillari molto sottili, molli, tutte uniformi; ligula \pm parzialmente disintegrata in fibre sottili, molli non o poco arruffate. Picciolo gracilissimo, lungo 7-12 cm., largo $1.5 \mathrm{~mm}$., subbiconvesso, \pm scabridulo-striato. Lamina molto piccola, lunga $8-10 \mathrm{~cm}$., rigidula, cartacea, divisa sino alla base in soli 2-4 segmenti, digitati, 2-3plicato-costulati, nell' insieme fra tutti con sole 7-9 costole primarie superiori, acuti in basso, leggermente ristretti all'apice, molto inegualmente inciso-dentati a denti acutiusculi, di sotto più pallidi che di sopra, senza squamule brune (sempre?); margini finamente serrulati; costole superiori scabridule; venule transverse conspicue.

Spadici nascenti eretti framezzo le fronde, presso a poco lunghi quanto $\mathrm{i}$ piccioli di queste, con parte pedunculare assai allungata compressa e che si divide all'apice in soli $2-4$ ramoscelli, i quali portano pochissimi fiori sparsi; spate primarie angustissime, larghe $2-3 \mathrm{~mm}$., terminate in lembo stretto, acuminato, giungente sino a circa la metà della parte peduncolare dello spadice. Gli esemplari da me visti portano solo pochi fiori feminei trapassati.

Habitat. - Vallata del Me-Kong nel Laos inferiore a Lakôn (o Lacòne), Thorel n. 3099 nell' Erb. di Parigi.

Osservazion. - Affine alla Rh. laosensis, ma distinta per le sue piccole dimensioni, per le piccolissime fronde digitate, che nell'insieme hanno sole 7-9 costole primarie superiori, e si dividono in soli $2-4$ segmenti ; per gli spadici con assai lunga parte pedunculare sporgente dalie angustissime spate. 


\section{Calamus acanthophyllus Becc. sp. n.}

Parvus, subacaulis, caespitosus, vaginis non flagelliferis in ventre apertis. Folia erecta, rigida, 50-80 $\mathrm{cm}$. longa (non cirrifera),; petiolo longiusculo; segmentis utrinque 12-14, inequidistantibus, rigidis et spisse papyraceis, utrinque pallide virentibus vel subglaucescentibus, linearibus, apice \pm attenuatis, acutis et subpungentibus vel interdum obtusiusculis, in costa media et ad margines saepius conspicue spinulosis, intermediis $15-25 \mathrm{~cm}$. longis, 6-10 mm. latis, summis radiato-digitatis brevioribus, duobus terminalibus basi plus minusve connatis. Spadices masculi et foeminei similes, erecti, simplicissimi; parte axili indivisa, rigida, apice 4-8 spicas floriferas ferenti; spathis arctissime vaginantibus; spicis rigidis, $2-8 \mathrm{~cm}$. Iongis; floribus disticis, utrinque 8-10; floribus masculis anguste ovoideis, 4-5 $\mathrm{mm}$. longis; floribus foemineis ovoideis, $3 \mathrm{~mm}$. longis, calyce profunde 3-lobo ; corolla calyce subtriplo longiori; perianthio fructifero breviter pedicelliformi. Fructus ovoidei vel subovoidei, minute acuteque conice rostrati, 12-15 $\mathrm{mm}$. longi, 9-12 $\mathrm{mm}$. lati; squamis superficialiter in medio sulcatis, stramineis et anguste nigro-marginatis, apice acutis; semine elliptico, paullisper compresso, $7 \mathrm{~mm}$. longo, 5 $\mathrm{mm}$. lato, $4 \mathrm{~mm}$. spisso; albumine aequabili; embryone basilari.

Habitat. - Nella Cocincina sul fiume Ubon, raccolto dal Dott. Thorel durante la spedizione del Me-kong (1866-68). Nel Laos (Massic, Erb. di Parigi).

Osservazioni. - 亡́丶 una specie singolare ed aberrante, ma che nondimeno si può raggruppare col C.erectus $\theta$ col C. dongnajensis.

Non sembra mai scandente; non ha flagelli alle vagine; ha foglie non cirrifere e spadici semplicissimi non flagelliferi, maschi e feminei fra loro similissimi. In alcuni 
esemplari le foglie hanno i segmenti spinosissimi sui margini e sulla costa mediana, ma alle volte questa è quasi inerme ed $i$ margini portano solo qualche spina in qua e là.

\section{Calamus Balansaeanus Becc. sp. n.}

Gracillimus, erectus (vel demum scandens ?), caudice vaginato $8 \mathrm{~mm}$. diam., circ. $1.5 \mathrm{~m}$. longo; frondium ecirrosarum (circ. $65 \mathrm{~cm}$. long.) segmenta inaequidistantia vel irregulariter fasciculata, anguste lineari-lanceolata, superne sensim in acumen tenue barbatum attenuata, costulis 3 tonuibus in facie superiori spinulosis percursa, subtus in costa media tantum setulosa $\nabla e l$ omnino nuda; venulis transversis conspicuis discoloribus; marginibus minutissime spinulosis; segmenta intermedia $15-17 \mathrm{~cm}$. longa, 10-13 mm. lata ; superiora sensim minora, duo terminalia basi omnino libera. Spadix foemineus gracilis, elongatus, filiformis, apice flagello tenui aculeolato terminatus; inflorescentiis partialibus paucis, erectis, remotis, $10-12 \mathrm{~cm}$. longis, spiciformibus, angustis, strictis, in earum parte basilari spiculas floriferas parvas, superne flores ternos geminatosve, et in parte apicali solitarios, ferentibus ; spathae secundariae et spathellae leves, oblique infundibuliformes, apice marcescentes; involucrophoro oblique cupulari-cyatiformi; involncro subauriculaeformi ; floris neutri areola concavo-aedicolaeformi. Fructus globosus, breviter rostratus, circ. $1 \mathrm{~cm}$. diam.; squamis per orthostichas 21 ordinatis, stramineis et anguste nigro-marginatis, in medio obsolete sulcatis, apice obtusiusculis; semine irregulariter globoso, areolis nonnullis concaviusculis notato, $7 \mathrm{~mm}$. diam., albumine aequabili; embryone basilari.

Habitat. - Nel Tonkino a Than Mö̈, nelle macchie. Balansa, 3 Marzo 1886, n. 517 nell'Erb. di Parigi.

Osservazioni. - $\dot{\mathrm{E}}$ certamente affine al $C$. Henryanus Becc., dal quale si distingue per lo spadice con infiorazioni 
parziali molto semplici, distintamente spiciformi, portanti piccole spighette nella parte bassa e i fiori terni, geminati o solitarii in quella alta, tutti appressi alla parte assile in modo da formare una sola spiga densa ed angusta. L'involucro ba poi l'areola che porta il fiore femineo in forma di nicchia o di edicola e manca del piccolo pedicello che sorregge il fiore neutro nel $C$. Henryanus.

Come il C. Henryanus mostra della affinità col C. leptospadix.

Calamus Bonianus Becc. sp. n.

Gracilis, alte scandens, caudice vaginato digiti crassitiae; folia (non cirrifera) parva, $48-55 \mathrm{~cm}$. longa; petiolo brevi; segmentis utrinque 10-14, distincte fasciculatis, lanceolatis, anguste elliptico-lanceolatis vel oblanceolatis, $12-20 \mathrm{~cm}$. longis, $2-3 \mathrm{~cm}$. latis, basi acutiusculis, superne breviter et saepe abrupte-acuminatis, subtiliter papyraceis, tenuissime 3-5-7-costulatis, utrinque nudis vel in costa media tantum supra parce spinulosis; venulis transversis numerosissimis tenuibus sed prominulis; marginibus patule spinuloso-ciliatis; segmentis 2 terminalibus fere usque ad medium unitis. Spadix masculus elongatus, ultradecompositus, spicis arcuato-subscorpioideis, $1-2 \mathrm{~cm}$. longis, 15-16 flores (in totum) biseriatim assurgentes ferentibus; floribus masculis 3-5 mm. longis, ovoideo-ellipticis, acutis. Spadix foemineus duplicato-ramosus, $1.5 \mathrm{~m}$. long., apice flagellifer, circiter 8 inflorescentias partiales ferens; spathis primiariis tubulosis, superne paullo ampliatis, ibique lexiuscule vaginantibus; spicis arcuatis, patentibus vel deflexis, majoribus $6-8 \mathrm{~cm}$. longis; foribus bifariis, utrinque numerosis; spathellis basi tubulosis, superne ampliato-infundibuliformibus ; involucrophoro sessili, plano, discoideo-orbiculari ; involucro involucrophoro simili ; floris neutri areola punctiformi ; perianthio fructifero distincte pedicelliformi. Fructus parvi, sphaerici, 4 mm. diam., abrupte rostrati ; squamis per orthostichas 21 ordinatis, stramineis, apice rubellis, in medio sulcatis. 
Habitat. - Nel Tonkino occidentale. Scoperto dal Padre Bon a Jên Cu. (N. ${ }^{\circ}$ 1211, esempl. con frutti) ed a Tai-Kêuh (n. ${ }^{\circ}$ 3549, spadice maschio). Negli Erb. di Parigi e De Candolle. Nome volgare \& Cây Mai ".

Osservazioni. - $\dot{\mathrm{E}}$ molto prossimo al C. tetradactylus Hance, dal quale si distingue per le infiorazioni parziali più dense, con maggior numero di spighette e queste più fornite di fiori; per l' involucroforo sessile e non pedicellato, ed a quanto sembra anche per il frutto più piccolo.

\section{Calamus cambojensis Becc. sp. n.}

Gracillimus, scandens, caudice vaginato $6-8 \mathrm{~mm}$. diam.; vaginis nonnullis flagelliteris; folia (non cirrifera) brevia $35 \mathrm{~cm}$. longa; petiolo brevi; segmentis paucis (utrinque 6-7), inaequidistantibus, remote fasciculatis, anguste ellipticis, utrinque aequaliter attenuatis, tenuiter papyraceis, costulis 5 tenuibus, utrinque nudis, percursis, vel in costa media supra sparse spinulosis; venulis transversis approximatis, praecipue in pagina superiori conspicuis; marginibus patule spinuloso-ciliatis; segmentis intermediis $10-12 \mathrm{~cm}$. longis, 2-3 cm. latis, duobus terminalibus basi plus minusve connatis. Spadix foemineus flagelliformis, tenuis, $0.8-1 \mathrm{~m}$. longus, flaccidus, foliis longior, simpliciter decompositus; inflorescentiis partialibus 3-4, remotis, gracilibus, utrinque 5-7 spicas ferentibus; spathis primariis tubulosis, arcte vaginantibus, angustis, elongatis; spicis arcuatis, plus minusve deflexis, 2-3.5 cm. longis; floribus disticis utrinque 5-8; spathellis late infundibuliformibus; involucrophoro sessili concavo; involucro suborbiculari depresse cupulari; floris neutri areola semilunari; floribus foemineis ovoideis; floribus neutris conspicuis foemineis simillimis, attamen paullo minoribus. Caetera desunt.

Habitat. - Nel Camboge. Gli esemplari sui quali la specie è stabilita vennero inviati dal sig. Gourgaud all'Espo- 
sizione coloniale di Marsiglia del 1906 e presentemente si trovano nell'Erb. del Museo di Parigi; non è indicata la località precisa dove vennero raccolti e portano il nome indigeno "Phdau-San ".

Osservazioni. - Si avvicina al C. tetradactylus ed al C. Bonianus per le foglie, specialmente per la disposizione dei segmenti; ma lo spadice femineo è più flaccido e con le spate più anguste $\theta$ più strettamente guainanti. I segmenti hanno le venule transverse anche più fitte che nel C. Bonianus.

Per le foglie rassomiglia molto al C. tetradactylus Hance, di Hong-Kong, ma in questo le spate primarie sono lassamente guainanti ed alquanto dilatate in alto, mentre quelle del C. cambojensis sono angustissime e molto strettamente abbraccianti l'asse dello spadice.

Sembra riferibile al $C$. cambojensis un esemplare portante uno spadice maschile, raccolto dal Dott. Thorel (n. ${ }^{\circ} 2974$, Erb. di Parigi) sulle montagne a Bassac (Me-Kong). Il tronco e l'armatura delle guaine è come negli esemplari tipici. Le foglie sono un poco più grandi che negli esemplari feminei, sono lunghe $45 \mathrm{~cm}$, hanno il picciolo un poco più lungo $(3-4 \mathrm{~cm}$.) e qualche segmento di più, 8 per parte, più il terminale che è bipartito, i segmenti sono un poco più grandi, gli intermedii lunghi sino $15-20 \mathrm{~cm}$. e larghi 27-30 mm., del resto esattamente $\operatorname{come}$ di già descritti. Lo spadice maschio è molto semplice, gracillimo, flagelliforme, sottilissimo, con pochissime e piccole infiorazioni parziali; spate angustissime, molto strettamente guainanti; spighette pettiniformi, lunghe $15-25 \mathrm{~mm}$. e con $6-8$ fiori orizzontali per parte; fiori lunghi $4 \mathrm{~mm}$., engusti, tereti, acuti; calice latamente 3-dentato, fortemente costulatostriato; corolla circa 3 volte più lunga del calice. 


\section{Calamus scutellaris Becc. sp. n.}

Ut videtur robustus, caudice et foliis ignotis. Spadix foemineus amplns, dense duplicato-ramosus, non cirrifer; inflorescentiis partialibus $20-30 \mathrm{~cm}$. longis (et interdum ultra?), in ramulos paucos approximatos divisis; spathis secundariis brunneis, inermibus, kasi tubulosis, membranaceis, superne laceris, laciniis valde elongatis et spicarum apicem fere attingentibus; ramulis brevibus arcuatis, spicas floriferas 4-6 approximatas brachiatim ferentibus; spathis tertiariis brevissime cyathiformibus, truncatis, integris, levibus; spicis sinuosis, vermiformibus, 7-9 cm. longis, parte axili tereti, $3 \mathrm{~mm}$. diam.; floribus exacte disticis, utrinque 20-25; spathellis breviter infundibuliformibus; involucrophoro breviter cupulari, subpedicellato; involucro conspicuo, orbiculari, integro, concavo-pateriformi; floris neutri areola conspicua, late ovoidea, concaviuscula. Perianthium fructiferum explanatum. Fructus parvi, ovoidei, breviter acuteque rostrati, $12 \mathrm{~mm}$. longi, $8 \mathrm{~mm}$. lati, squamis per orthostichas 21 ordinatis, brunneis, subsquarrosis, in medio non sulcatis, margine fimbriato ferrugineo, apice acuto; semine late ovoideo, utrinque rotundato; albumine aequabili; embryone subbasilari.

Habitat. - Le porzioni di spadici con frutti maturi, che rappresentano tutto quanto si conosce di questa specie, vennero comprate dal $R$. Padre Bon sul mercato di Thanhhoa nel Tonkino orientale, nell'Ottobre 1892 (u. 5743 nell'Erb. di Parigi). Probabilmente i nativi si servono dei frutti di questo Calamus come condimento, o per masticare in sostituzione della. Noce di Areca.

Osservazroni. - Appartiene al gruppo XV (A) della mia Monografia, ma non sembra affine ad alcune delle specie conosciute, eccettuato forse il C. Scipionum. E particolarmente notevole per l'involucro del fiore femineo, che allorchè sono caduti i frutti è molto conspicuo ed ha la forma 
di una tazza poco profonda. I suoi piccoli frutti sono pure notevoli per le squame brune leggermente squarrose, non solcate per il lungo, e con una frangia di peli ferruginosi sui margini.

\section{Plectocomiopsis floribundus Becc. sp. n.}

Frondium vaginis spinis gracilibus basi confluentibus et oblique seriatis vel pectinatis, armatis; petiolo brevi; segmentis per greges remotiusculos distincte approximatis, anguste lanceolatis, apice acuminato-subulatis, utrinque virentibus, subtus punctulatis, majoribus $20-30 \mathrm{~cm}$. longis, 2.5-3 cm. latis ; spadice masculo amplo, laxe ramoso, spicis scorpioideis, $8-10 \mathrm{~mm}$. longis ; floribus congestis non valde numerosis, per series 2 assurgentes ordinatis, ovoideis, acutis, obsolete trigonis, $3 \mathrm{~mm}$. longis; calyce membranaceoscarioso, profundissime trilobo ; corolla calyce $1 / 3$ vel $1 / 2$ longiori, in segmenta elliptico-navicularia, tenuiter coriaceoscariosa fere usque ad basin partita; staminum filamentis basi connatis, superne latiuscule linearibus, abrupte introflexis; antheris erectis, elongato-sagittatis; ovarii rudimento parvo papillaeformi. Coetera desunt.

Habitat. - Un esemplare con fiori maschi è stato raccolto nel Camboge da Gourgaud, con la sola indicazione «Pfdau-chno » (probabilmente il suo nome indigeno). Allo stato sterile è stato pure riportato dalla Cocincina dal Dott. Thorel, ma senza indicazione precisa di località.

Osservazioni. - Sembra assai affine al Plectocomiopsis paradoxus Becc. (Cal. paradoxus Kurz), ma in questo, sebbene i segmenti siano inequidistanti non sono cosi distintamente riuniti in gruppi come sono quelli del $P$. floribundus; anche i fiori maschili di questo sono di un terzo più piccoli di quelli del $P$. paradoxus, più angusti e più acuminati, e lo spadice è più diffuso. 


\section{Plectoconica Plerreana Becc. sp. n.}

Inter minores; frondium supremarum segmentis inaequidistantibus, lineari-lanceolatis, utrinque virentibus, subtus vix pallidioribus; fructibus sphaericis, abrupte rostratis, circ. $2 \mathrm{~cm}$. diam.; squamis per $42-44$ orthostichas ordinatis, squarrosis, apice valde producto acuto, ciliato, recto, minime recurvo ; semine globoso, nonnihil depresso, $14-15 \mathrm{~mm}$. lato, 11-11.5 m. alto; perianthio fructifero explanato, sepalis delthoideis acutis, corollae segmentis lanceolatis, acuminatis, calyce subtriplo longioribus; antheris sterilibus sagittatis.

Habitat. - Nel Camboge sui monti Cam-chây nella Provincia di Kampuh, all' altezza di 900 m. sul livello del mare (Pierre nell'Erb. di Parigi). Un altro esemplare, apparentemente della medesima provenienza, si trova nell'Erb. del "British Museum 》 e faceva parte dell'Erbario di Hance (n. ${ }^{\circ}$ 19241).

Osservazioni. - É una delle più piccole specie, delle dimensioni della Plectocomia Muellerii, alla quale rassomiglia anche per i frutti; ma quelli della P. Pierreana hanno le squame che si terminano in una punta dritta $\theta$ non come nella $P$. Muellerii ripiegata in dietro; inoltre la punta non si prolunga in lunghe setole. Si distingue dalla $P$. Muellerii anche per i segmenti delle fronde superiori più stretti $\theta$ per le antere degli stami sterili nel fore femineo sagittate, mentre sono lineari nella $P$. Muellerii. 
ENUMERAZIONE delle specie di Palme sino a qui note come crescenti selvatiche nell'Indo-China o che vi sono estesamente coltivate.

\section{ARECEAE.}

\section{Areca Catechu Linn.}

Coltivata.

Areca laosensis Becc. Vedi sopra a p. 191.

Areca triandra Roxb. Hort. Beng. 68.

Cocincina merid. sul monte Ponglu a $150 \mathrm{~m}$. sul livello del mare presso Binh-Thuan (Pierre ${ }^{0}{ }^{0} 4849$ ) e sui monti Dinh presso Baria (Pierre col med. n. ${ }^{\circ} 4849$ ).

Pinanga cochinchinensis Bl. in Bull. Néerl. 1838, 65; Becc. Malesia III, 143. - Areca sylvestris Lour. Fl. Coch. ๖̌68; edit. Willd. 696.

Cocincina meridionale a Boo-chiang, Prov. di Bien-hoa (Pierre n. ${ }^{\circ}$ 1876).

Pinanga Duperreana Pierre mss. ex Becc. Malesia III, 144.

Camboge, monti Kuang-Repen, Prov. di Jpong (Pierre n. ${ }^{\circ} 4851$ e 4853$)$.

PInanga baviensis Becc. Vedi sopra a p. 193.

Oncosperma fllamentosa Blume, Rumphia II, 97, t. 82, 103. O. cambodianum Hance in Journ. of Bot. 1876, p. 261.

Isola Phu-Quoc nel Golfo del Siam (Pierre n. ${ }^{0}$ 1859). 
Wallichia gracilis Becc. sp. n. Vedi sopra a p. 211.

Arenga saccharifera Labill. in Mém. Inst. Fr. IV, 209. Borassus Gumutus Lour. Fl. Coch. II, 619.

Cocincina merid., sui monti Dinh, Prov. di Baria (Pierre n. ${ }^{0}$ 44) subspontanea, ed assai spesso coltivata.

Didymosperma caudata W. et Dr. Vedi sopra a p. 200, 201.

- - var. stenophylla Becc. Vedi sopra a p. 206.

_ - var. tonkinensis Becc. Vedi sopra a p. 208.

Caryota mitis Lour. Fl. Coch. II, 569. - C. sobolifera Wall. Cat. 8594; Mart. Hist. Nat. Palm. III, 194, t. 107, f. 2.

Vicinanze di Saigon (Pierre n. ${ }^{0} 1107$ ) ed a Dinh, Prov. di Baria, Cocincina meridionale (Pierre n. ${ }^{0}$ 105).

Caryota monostaclya Becc. sp. n. Vedi sopra a pag. 196. Nipa fruticans Wurmb. in Verh. Bat. Genootsch. 1, 349.

Non ho visto esemplari di questa Palma provenienti dall'Indo-China, dove certamente cresce nei luoghi inondati delle coste meridionali.

\section{PHOENICEAE.}

Phoenix. Roebelinil O'Brien in Gard. Chr. 26 Oct. 1889, p. 475 , f. 68 , et 28 Dec. 1889 , p. 750 - Ph. pusilla Lour. (non Gaertn.) Fl. Coch. II, 614? - Ph. Lourerii Kunth, Emum. pl. III, 257 ?

Questa Palma, che è ora estesamente coltivata in Europa nelle serre ed in pien'aria in molte parti della Regione mediterranea, è stata scoperta sulle sponde rocciose del 
Mekong nel Laos. A questa specie indubbiamente appartengono gli esemplari delle Piante del Tonkino di Balansa portanti i numeri 4471 (esemplare fruttifero) e 4877 (sole fronde), ma senza indicazione di località precisa. Nella "Malesia " (v. III, p. 382), io ho creduto di potere identificare la Ph. pusilla di Loureiro con una Phoenix raccolta da Pierre nella Cocincina, che certamente rientra nel gruppo della Ph. humilis; però data la dimensione di 4 linee (= a circa $1 \mathrm{~cm}$.) assegnata da Loureiro al frutto della sua Ph. pusilla, mi sembra molto probabile che alla $P h$. Roebelinii, piuttosto che alla $P h$. humilis, corrisponda la specie di Loureiro.

Secondo questa identificazione la Phoenix Roebelinii dovrebbe assumere il nome di $P h$. Loureiri, essendo quello di Ph. pusilla stato precedentemente adoprato per una specie del Ceylan.

Loureiro ha lasciato scritto che la sua Ph. pusilla abita in luoghi sassosi lungo $i$ ruscelli sui monti Côu-nut a sei leghe di distanza da Hue. Con questa indicazione non dovrebbe esser difficile di rintracciare questa Palma nella località classica $\theta$ cosi risolvere la questione della sua precisa nomenclatura.

Phoenix humilis Royle, Illustr. of the Him. mount. I (1839), 8, 14, 394, 397, 399 ; Becc. Malesia, III, 373, t. XLIV, II.

Camboge, sul monte Kuang Repen: Harmand n. 4832 in Erb. Pierre.

Gli esemplari di Pierre corrispondono esattamente a quelli della Ph. humilis che cresce nell'Assam e nella Birmania.

Phoenix paludosa Roxb. Hort. Beng. 75.

Cocincina merid. a Beutré (Pierre n. ${ }^{0} 4835$ ).

\section{Phoenix sp.}

Sulle sponde del Mekong, a Stung-Streng, il D. Thorel ha raccolti degli esemplari sterili di una Phoenix apparte- 
nenti, a quanto sembra, ad una specie differente dalle precedenti.

\section{CORYPHEAE.}

Corypha Linn.

Non ho visto esemplari dell'Indo-China di alcuna specie di questo genere di Palme, che mi sembra ben difficile che non sia rappresentato in quella regione.

Iicuala spinosa Wurmb. in Verh. Bat. Genootsh. II, 469. - L. spinosa var. cochinchinensis et var. brevidens Becc. Malesia, III; - Corypha pilearia Lour. Fl. Cochinch. I, 265.

Cocincina merid. a Song-lu nella Prov. di Bien-hoa e sul monte Dai nella Prov. di Chau-doc. A Bencat lungo il fiume di Saigon. Nell' isola di Phu-Quoe nel Golfo del Siam. (Pierre nell'Erb. di Parigi).

La Licuala spinosa è risultata adesso una specie talmente diffusa e così poliforma che la distinzione in varietà del valore di quelle rammentate diventerebbe infinita.

Inicuala paludosa Griff. in Calc. Journ. Nat. Hist. V, 233, et Palms Brit. Ind. 118, t. 221, $A, B, C$.

Isola di Phu-Quoc nel Golfo del Siam (Pierre n. ${ }^{\circ} 4839$ ).

Iicuala tonkinensis Becc. sp. n. Vedi sopra a p. 214.

Iicuala calciphila Becc. sp. n. Vedi sopra a p. 216.

Iicuala fatua Becc. sp. n. Vedi sopra a p. 218.

Rhapis micrantha Becc. sp. n. Vedi sopra a p. 220.

Rhapis laosensis Becc. sp. n. Vedi sopra a p. 225.

Rhapis subtilis Beci. sp. u. Vedi sopra a p. 227. 
Iristona cochinchinensis Mart. Hist. nat. Palm. III, 319; Becc. Malesia III, 69. - L. spectabilis Griff. in Calc. Journ. Nat. Hist. $\nabla, 336$, et Palms Brit. Ind. 130, t. CCXXVI. G. - Corypha Saribus Lour. Fl. Cochin. I, 212.

Cocincina merid. a Thuduc ed a Song-lu, Prov. di Bienhoa (Pierre, n. ${ }^{\circ}$ 4837). Nel Tonkino meridionale a Lang Doi (Père Bon n. 1549: nome vol. «Cay-Co ») ed a Vau xa (n..$^{\circ} 3276$ ) e n. ${ }^{\circ}$ 6184: senza località precisa. Pure nel Tonkino a Tu-Phap (Balansa $\mathrm{n}^{\circ} 4336$ ). Le foglie servono a cuoprire capanne ed a fare $i$ grandi cappelli annamiti.

\section{BÒRASSINEAE.}

Borassus flabellifer Linn. sp. pl. p. 1187. - B. tunicata Lour. Fl. Cocinch. edit. Willd. p. 760.

Dalle descrizioni dei viaggiatori sembra molto diffuso nell'alto Mekong.

\section{COCOINEAE.}

Cocos nuclfera Linn.

Estesamente coltivato.

\section{LEPIDOCARYEAE.}

Calamus dongnaiensis Pierre mss. ex Becc. in Rec. Bot. Surv. Ind. ii, 189 ; Becc. in Ann. R. B. G. Calc. XI, 133 , t. 8.

Cocincina merid, a Chiao-xhan (Pierre n. ${ }^{\circ} 4829$ ).

Calamus acanthophyllus Becc. sp. n. Vedi sopra a pag. 229.

Calamus rudentum Lour. Fl. Cochinch. I. 209; Becc. in Ann. R. B. G. Calc. XI, 139, t. 13.

Cocincina merid. a Dian-lau-me, Prov. Bien-hoa (Pierre n. ${ }^{\circ} 4846$ ); a Tan Kiyen sul fume Dongnai (Pierre n. ${ }^{\circ} 4845$ ) 
e presso il monte Pong-lu, Prov. Bin-Thuan (Pierre senza n. $^{\circ}$ ).

Calamus Balansaeanus Becc. sp. n. Vedi a pag. 230.

Calamus dioicus Lour. Fl. Cochinch. 1, 210 ; Becc. in Ann. R. B. G. Calc. XI, 195, t. 51.

Monti Chiao-xhoin (Pierre n. ${ }^{0}$ 4834).

Calamus viminalis Willd., cochinchinensis Becc. in Ann. R. B. G. Calc. XI, 207, t. 57.

Cocincina meridionale : monti Kuang Repen, Prov. Ipong (Pierre n. ${ }^{\circ} 4848$ ); a Songlu, Prov. di Bien-hoa, e sulle montagne Dây, Prov. Chaudoc (Pierre senza n. ${ }^{\circ}$ ).

Calamus tenuis Roxb. Fl. Ind. III, 780 ; Becc. in Ann. R. B. G. Calc. XI, 262, t. 94. - C. amarus Lour. Fl. Cochinch. I, 210?

Cocincina meridionale sui monti Dinb (Mu-xoai) presso Baria (Pierre senza n..$^{\circ}$.

Calamus Godefroyi Becc. in Ann. R. B. G. Calc. XI, 267. t. 96.

Cocincina merid. Gran lago presso Siem Reap (Godefroy-Leboeuf in Herb. Kew). Ad Hong-Kay (Thorel, spediz. del Mekong, senza n. ${ }^{\circ}$ ).

Calamus tonkinensis Becc. in Anv. R. B. G. Calc. XI, 275 , t. 100 .

Tonkino a Taukeiun presso Zuang-yen (Balansa n. ${ }^{\circ} \mathbf{5 1 6}$ in Herb. Petrop. et Kew.).

Calamus salicifolius Becc. in Rec. Bot. Surv. Ind. II, 206, et in Ann. R. B. G. Calc. XI, 279, t. 103.

Cocincina merid. a Tong-Keon presso Saigon (Pierre n. ${ }^{\circ} 4853$ ); pure presso Saigon (Germain, Herb. Delessert, $\theta$ Godefroy-Leboeuf in Herb. Kew). 
Calamus salicifolius var. leiophyllus Becc. in Ann. R. B. G. Calc. XI, 281.

Camboge a Campong Chouong (Otto Kunze, n..$^{\circ} 3995$, in Herb. Kew).

Calamus Bonianus Becc. sp. n. Vedi sopra a p. 231.

Calamus cambojensis Becc. sp. n. Vedi sopra a p. 232.

Calamus Guruba Ham. in Mart. Hist. nat. Palm. III, p. 206 (edit. 2. ${ }^{a}$ ) et 330 ; Becc. in Ann. R. B. G. Calc. XI, 299, t. 144 e 115.

Sponde del Mekong a Bassac, fiume Ubon (Thorel senza $\mathrm{n}^{0}$ in Herb. Paris).

Calamus Bousigonil Pierre mss. ex Becc. in Rec. Bot. Surv. Ind. II, 209, et in Ann. R. B. G. Calc. XI, 343, t. 139.

Cocincina meridionale a Baria nei monti Ma-xoai (Pierre n. ${ }^{\circ}$ 4836).

Calamus scutellaris Becc. sp. n. Vedi sopra a p. 234.

Calamus palustris (Griff.) cochin-chinensis Becc. in Rec. Bot. Surv. Ind. II, 211, et in Ann. R. B. G. Calc. $\mathrm{XI}, 405$, t. 175 .

Cocincina merid. sui monti Dinh presso Baria (Pierre n. ${ }^{\circ} 48 i$ ). Camboge sui monti Kuang Repen, Prov. Ipon (Pierre senza $1 . .^{\circ}$ ).

Calamus platyacanthus Warb. Mss. ex Becc. in Ann. R. B. G. Calc. 442 , t. 198.

Tonkino, Monte Bavi (Balansa n. ${ }^{\circ} 4360$ ).

Daemonorops Pierreanus Becc. in Rec. Bot. Surv. Ind. II, 220, et in Ann. R. bot. Gard. Calc. XII, t. 9 (ined.) Camboge. Monti Kuang Repen, Prov. Ipong (Pierre n. 4859$)$. 
Esistono altri esemplari incompleti di Daemonorops dubbiamente riferibili al $D$. Pierreanus, o forse appartenenti a specie affini, raccolti da Pierre nella Cocincina merid. a Toikuyen, Prov. Bien-hoa, e dal Dott. Thorel pure in Cocincina, (n. 929 in Herb. Paris).

Zalacca Reinw.

Di questo genere non ho visto esemplari di specie selvatiche o coltivate in Indo-China, dove mi sembra ben difficile che nell'un modo o nell'altro non sia rappresentato.

Zalaccella Farmandi Becc. in Ann. R. B. G. Calc. XI, 496 , t. 229 (sub Calamo).

Cocincina meridionale a Thulet-Thay (Harmand $n . .^{\circ} 1198$ in Herb. Pierre).

Plectocomia Pierreana Becc. sp. n. Vedi sopra a p. 236.

Pletocomiopsis flortbundus Becc. sp. n. Vedi a p. 235.

\section{Korthalsia lacinfosa Mart. ?}

Riferisco dubitativamente a questa specie alcuni esemplari sterili di una Korthalsia raccolta da Pierre nel Camboge sul Monte Keerev, Prov. Samrong-tong (n. ${ }^{\circ} 4860$ ) ed a Songlu, Prov. Bien-hoa (n. ${ }^{0}$ 1878).

Forse appartengono ad una seconda specie di Korthalsia altri esemplari, pure sterili, raccolti da Pierre a Bao-Chiang in Cocincina (.$^{\circ}$ 1877). Questi sono particolari per i segmenti oblanceolato-cuneati o subromboidali, distintamente coperti da un indumento bianco-farinoso e morbido nella pagina inferiore, carattere invero che talora sparisce o si attenua nelle fronde di pianta adulta.

Pigafetta flaris (Mart.) Becc. Malesia, I, 91. - Calamus Kunzeanus Becc. in Ann. R. B. G. Calc. XI, 490, t. 226, f. IV.

Camboge (Otto Kunze in Herb. Berol.). 
Metroxylon Rottb.

Non ho visto rappresentati di questo genere provenienti dall'Indo-China, dove senza alcun dubbio l' una o l'altra o forse ambedue le specie che somministrano il Sagu si debbono trovare, o coltivate o semispontanee.

SPECIE DUbBie.

Rhapis ? cochinchinengis Mart. Hist. nat. Palm. III, 254. - Chamaerops cochinchinensis Lour. Fl. Coch. II, 657 ; edit. Willd. II, 808.

Palma molto dubbia, perchè stando alla descrizione di Loureiro per la corolla monopetala si potrebbe ritenere realmente per una Rhapis, mentre per i piccioli (stipiti) spinosi e per le fronde che si dice si adoprano « ad tegendos domos et construenda pallia solem et pluviam repulsura » sembrerebbe trattarsi di una Licuala o di una Livistona; giacchè non vi è foglia di Rhapis sino a qui conosciuta che abbia dimensioni tali da poter servir per tale oggetto.

Il nome volgare di "Cay Lui ", assegnato da Loureiro alla sua Chamaerops cochinchinensis in realtà corrisponde a quello che il Padre Bon assegna alla Rhapis che io ho descritto col nome di Rhapis micrantha. $\dot{\mathrm{E}}$ possibile che la specie sia stata fatta sopra i fiori di una Rhapis e le frondi giovani di Livistona cochinchinensis.

Fra i Calamus descritti da Loureiro vi sono i seguenti che non è ancora stato possibile d'identificare. (Si veda in proposito: "The species of Calamus 》 in Annals of the Royal Botanic Garden, Calcutta * vol. XI, p. 38).

Calamus petraeus Lour. - Forse identificabile con la Plectocomia Pierreana Becc.

Calamus verus Lour. - É probabilmente una specie di Daemonorops.

Calamus amarus Lour. - Forse corrisponde al Calamus tenuis Roxb. 